\title{
MULTIVALUED SOLUTIONS TO THE EIKONAL EQUATION IN STRATIFIED MEDIA
}

\author{
BY \\ S. IZUMIYA (Dept. of Mathematics, Hokkaido University, Sapporo 060, Japan), \\ G. T. KOSSIORIS (Institute of Applied and Computational Mathematics, FORTH, 71110 \\ Heraklion, Crete, Greece and Dept. of Mathematics, Univ. of Crete, Heraklion, Crete, Greece), \\ AND \\ G. N. MAKRAKIS (Institute of Applied and Computational Mathematics, FORTH, 71110, \\ Heraklion, Crete, Greece)
}

\begin{abstract}
In the present paper we study the geometric properties of the multivalued solutions to the eikonal equation and we give the appropriate classification theorems. Our motivation stems from geometrical optics for approximating high frequency waves in stratified media. We consider the case of a fixed Hamiltonian imposed by the medium, and we present the geometric framework that describes the geometric solutions, using the notion of Legendrian immersions with an initial point source or an initial smooth front. Then, we study the singularities of the solutions in the case of a smooth or piecewise Hamiltonian in a boundaryless stratified medium. Finally, we study the singularities of the solutions in a domain with a boundary that describes the propagating field in a waveguide.
\end{abstract}

1. Introduction. In the analysis of wave propagation in inhomogeneous media the method of classical geometrical optics is often employed. Not only is it used to get a qualitative picture of how the waves propagate, but also to evaluate the fields quantitatively. However, geometrical optics fails either on caustics and focal points where it predicts infinite wave amplitudes (see Sec. 2), or in shadow regions (i.e., regions devoid of rays) where it yields zero fields. On the other hand, formation of caustics is a typical situation in underwater acoustics and seismology, as a result of multipath propagation from localized sources. Indeed, even in the simplest oceanic models and geophysical structures (see, e.g., Tolstoy and Clay [TC], Chapt. 5, and Cĕrvenỳ, et al [CMP], Chapt. 3 , respectively) a number of caustics occur, depending upon the position of the source

Received July 30, 1999.

2000 Mathematics Subject Classification. Primary 35Q99, 58C28.

This work has been partially supported by the TMR Contract FMRX-CT98-0234.

(C)2001 Brown University 
and the stratification of the wave velocities. From the mathematical point of view, formation of caustics and the related multivaluedness of the phase function, is the main obstacle in constructing global high-frequency solutions of the Helmholtz equation.

The qualitative study of the multivalued phase function is performed using the geometric techniques of singularity theory. The classification theory for general caustics and bifurcations of wave fronts has been originated by Hörmander [HO2], and Arnol'd and Zakalyukin (see, e.g., $[\mathrm{AVH}]$ ). However, their theory is generic and it describes a setting for a wide class of functions, but not the caustics and wavefronts corresponding to a fixed Hamiltonian function. The geometric framework for the study of caustics for a fixed Hamiltonian is given by Jänich [JA] and Wassermann [WA]. For the bifurcation of wavefronts, see Izumiya [IZ], Bogaevskir [BO]. If the Hamiltonian function is nonsmooth, the situation is rather complicated. The first attempt to study this case has been made by Kazarian [KA] and Myasnichenko [MY], but their framework is also too general for applications to a specific Hamiltonian. In the case of domains with boundary, rather different kinds of caustics may appear, namely the boundary caustics, which have been classified in lower dimensions by Scherbak [SC] (see also [TS1], [TS2]). These results will be described in Sec. 3 .

The problem of obtaining numerically the multivalued phase function has traditionally been handled by resolving numerically the characteristic field related to the eikonal equation (ray tracing methods); see, e.g., [CMP]. A considerable amount of work has been done recently on constructing the multivalued phase function by properly partitioning the propagation domain and using eikonal solvers (see, e.g., [BEN], [FEO], [SYM], $[\mathrm{ABG}])$. A different approach is based on kinetic formulation in the phase space, in terms of a density function that satisfies Liouville's equation. The technique used to capture the multivalued solutions is based on a closure assumption for a system of equations for the moments of the density ([RU], [BKP]).

On the other hand, given the geometry of the multivalued phase function, a number of local and uniform methods to describe wave fields near caustics have been proposed. The first type of methods are essentially based on boundary layer techniques as they were developed by Babich, Keller, et al (see, e.g., [BK], [BB], [GA]). The second type are those that exploit the fact that even if the family of rays has caustics, there are no such singularities for the family of the bicharacteristics in the phase space. This basic fact allows the construction of formal asymptotic solutions (FAS) that are valid also near and on the caustics. For this purpose two main asymptotic techniques have been developed. The first one is the Ludwig-Kravtsov method (or the method of relevant functions). This method starts with a modified FAS involving Airy-type integrals, the phase of which takes account of the type of caustics (see, e.g., [LU], [KR]). The second one is the method of canonical operator developed by Maslov (closely related to this are the techniques of Orlov's integrals [AND] and oscillatory spectral integrals [ARN1], [ARN2]). Maslov's method exploits the fundamental fact that the Hamiltonian flow associated with the bicharacteristics generates a Lagrangian manifold in the phase space, on which we can "lift" the phase function in a unique way (see, e.g., [MF], [MSS], [MA1], [MA2], [V1]).

In the present paper we study the geometric properties of the solution arising in problems of practical interest in an attempt to shed light on the geometry of the phase function 
that will attribute to the correct application of uniform methods that approximate the wave fields near caustics. We consider the case for a fixed Hamiltonian imposed by the stratified medium which may have a boundary. In Sec. 2 we briefly explain the role of the geometry of the multivalued solutions in geometrical optics. In Sec. 3 we present the geometric framework that describes the geometric solutions of the eikonal equation as it is given by the method of characteristics. We use the notion of Legendrian immersions in the case of an initial point source or an initial smooth front. In Sec. 3.1 we study the case of a smooth refraction index without boundary, and in Sec. 3.2 we present the classification of singularities for the latter case. In Sec. 3.3 we study the geometry of the phase function for a non-smooth refraction index without boundary that describes a boundaryless stratified medium with a weak interface. Finally, we study the geometry of the phase function in a domain with a boundary that describes the wave field in a waveguide.

2. Multivalued solutions in geometrical optics. In this section we present briefly the role of the multivalued solutions of the eikonal equation for the computation of high-frequency scalar wave fields of frequency $\omega$, in a medium with wave velocity $c(\mathbf{x})$, $\mathbf{x}=(x, z) \in D, D \subset \mathbb{R}_{\mathbf{x}}^{2}$ being the unbounded domain of propagation. The wave field is governed by the Helmholtz equation

$$
\Delta u+k^{2} \eta^{2}(\mathbf{x}) u(\mathbf{x}, k)=f(\mathbf{x}), \quad \mathbf{x} \in D,
$$

where $\eta(\mathbf{x})=c_{0} / c(\mathbf{x})$ is the refraction index, $c_{0}$ being the reference wave velocity, $k=$ $\omega / c_{0}$ is the wavenumber and $f$ is a compactly supported source generating the waves. We assume for the moment that $\eta \in C^{\infty}\left(\mathbb{R}_{\mathbf{x}}^{2}\right)$ and $\eta>0$.

For fixed $k>0$, there is, in general, an infinite set of solutions of 2.1 , and we need a radiation condition to guarantee uniqueness (cf. $[\mathrm{CK}]$ for scattering by compact inhomogeneities, and [WED] for scattering by stratified media). This condition is essentially equivalent to the physical fact that there is no energy flow from infinity, which in geometrical optics is translated to the requirement that the bicharacteristics must go off to infinity.

The asymptotic approximation of $u(\mathbf{x}, k)$ as $k \rightarrow \infty$ (i.e., for very large frequencies $\omega)$, constructed by applying the WKB procedure, is given by the asymptotic series

$$
u_{N}(\mathbf{x}, k)=e^{i k S(\mathbf{x})} \sum_{\ell=0}^{N}(i k)^{-\ell} A_{\ell}(\mathbf{x}),
$$

for $\mathbf{x}$ in a compact subset of $D \backslash \operatorname{supp} f$, where $N \rightarrow \infty$, and the phase $S$ and the amplitudes $A_{\ell}$ are real-valued functions in $C^{\infty}\left(\mathbb{R}_{\mathbf{x}}^{2}\right)$ (real geometrical optics).

Note that the asymptotic decomposition of scattering solutions when simultaneously $|\mathbf{x}|$ and $k$ go to infinity is a rather complicated problem, since, in general, the caustics of the Lagrangian manifold go off to infinity. This problem has been rigorously studied by Vainberg [V2], for the scattering of a plane wave, in the case where $D$ is a full neighborhood of infinity and $\eta=1$ for $|\mathbf{x}|>r_{0}, r_{0}$ being a fixed positive constant, and by Kucherenko $[\mathrm{KU}]$ for the case of a point source (i.e., $f(\mathbf{x})=\delta(\mathbf{x})$ ), under certain conditions of decay for $\eta(\mathbf{x})$ at infinity. 
The phase must satisfy the eikonal equation

$$
(\nabla S(\mathbf{x}))^{2}=\eta^{2}(\mathbf{x})
$$

for the phase function, and the amplitudes must satisfy the transport equations

$$
\begin{aligned}
& 2 \nabla S \cdot \nabla A_{0}+\Delta S(\mathbf{x}) A_{0}(\mathbf{x})=0, \\
& 2 \nabla S \cdot \nabla A_{\ell}+\Delta S(\mathbf{x}) A_{\ell}(\mathbf{x})=-\Delta A_{\ell-1}(\mathbf{x}), \quad \ell=1,2, \ldots
\end{aligned}
$$

The solution of the eikonal equation is constructed by the method of characteristics (see, e.g., [HO1], Chapt. VIII). The characteristics solve the Hamiltonian system in the phase space $\mathbb{R}_{\mathbf{x p}}^{4}$

$$
\begin{aligned}
& \frac{d \mathbf{x}}{d \tau}=\nabla_{p} H(\mathbf{x}, \mathbf{p})=\mathbf{p} \\
& \frac{d \mathbf{p}}{d \tau}=-\nabla_{\mathbf{x}} H(\mathbf{x}, \mathbf{p})=\eta(\mathbf{x}) \nabla \eta(\mathbf{x})
\end{aligned}
$$

associated with the Hamiltonian function

$$
H(\mathbf{x}, \mathbf{p})=\frac{1}{2}\left(|\mathbf{p}|^{2}-\eta^{2}(\mathbf{x})\right), \quad \mathbf{x} \in D, \mathbf{p} \in \mathbb{R}^{2} .
$$

The characteristics must satisfy the initial conditions

$$
\begin{array}{lll}
\mathbf{x}(0)=\mathbf{x}^{0}(\theta), & \mathbf{p}(0)=\mathbf{p}^{0}(\theta), & \theta \in I, \\
S(\mathbf{x})=S^{0}(\theta), & A_{l}(\mathbf{x})=A_{l}^{0}(\theta) & \text { for } \mathbf{x}=\mathbf{x}^{0}(\theta),
\end{array}
$$

on the initial manifold $U_{0}=\left\{\mathbf{x}=\mathbf{x}^{0}(\theta), \theta \in I \subset \mathbb{R}\right\}$. Here $\mathbf{p}^{0}(\theta)$ and $S^{0}(\theta), A_{l}^{0}(\theta)$ are given smooth functions and

$$
\left|\mathbf{p}^{0}(\theta)\right|^{2}=\left(\eta\left(\mathbf{x}^{0}(\theta)\right)\right)^{2}
$$

The projection $\{\mathbf{x}=\mathbf{x}(\tau, \theta), \tau \in \mathbb{R}, \theta \in I\}$ of the characteristics onto $\mathbb{R}_{\mathbf{x}}^{2}$ are the usual rays of geometrical optics. Assuming that $\mathbf{p}^{0}(\theta)$ is nowhere tangent to the initial manifold, the solution of the (non-characteristic) Cauchy problem (2.3), (2.9) for the eikonal equation is given by

$$
S(\mathbf{x}(\tau, \theta))=S^{0}(\theta)+\int_{0}^{\tau} \mathbf{p}(s, \theta) \frac{d \mathbf{x}(s, \theta)}{d s} d s,
$$

where the integral is calculated along the characteristics. The transformation

$$
(\tau, \theta) \mapsto(x(\tau, \theta), z(\tau, \theta))
$$

is one-to-one, provided that the Jacobian

$$
J=\left|\begin{array}{ll}
\frac{\partial x}{\partial \tau} & \frac{\partial x}{\partial \theta} \\
\frac{\partial z}{\partial \tau} & \frac{\partial z}{\partial \theta}
\end{array}\right|
$$

is nonzero. Although $J \neq 0$ for $\tau=0$, it does not necessarily remain nonzero for all $\tau$. Whenever $J=0,(\tau, \theta)$ may be non-smooth or multivalued functions of $(x, z)$, and the rays may intersect, touch, etc., and in general have singularities, although the characteristics never intersect in the phase space. Then, the phase function $S=S(x, z)$ may be a multivalued or a non-smooth function. 
The solution of the transport equation (2.4) for the principal amplitude $A_{0}$ on the ray is given by

$$
A_{0}(\mathbf{x}(\tau, \theta))=\frac{\alpha_{0}(\theta)}{\sqrt{J(\tau, \theta)}}
$$

where $\alpha_{0}(\theta)=A_{0}^{0}(\theta)$ is the amplitude at the point $\mathbf{x}=\mathbf{x}^{0}$ on the initial manifold, and $J(\tau, \theta)$ is the value of the Jacobian, so that $\mathbf{x}=(x(\tau, \theta), z(\tau, \theta))$ for the considered time. Note that the amplitude (2.12) is calculated by integrating the transport equation (2.4) in divergence form in a ray tube.

The locus of the points $\mathbf{x}=\mathbf{x}(\tau, \theta)$ at which $J(\tau, \theta)=0$, that is, the envelope of the family of the rays, is known as caustics. The amplitude $A_{0}$ blows up at these points, and therefore the WKB procedure fails to predict the correct amplitudes there. Fortunately, the caustics are only apparent singularities appearing when we apply the WKB procedure, and as we already mentioned in the Introduction, it is possible to construct uniform asymptotic expansions that remain finite on the caustics. The construction of such expansions, either in the form of Maslov's canonical operator (see [MF], [V1]) or in the form of the Kravtsov-Ludwig integral ([LU], [KR], [GS]), relies on the geometrical properties of the Lagrangian submanifold generated by the Hamiltonian system (2.6a), (2.6b).

The Kravtsov-Ludwig technique relies on the idea of obtaining global high-frequency solutions of the Helmholtz equation (2.1) by generalizing the asymptotic series (2.2) as an integral of the form

$$
u(\mathbf{x})=\left(\frac{i k}{2 \pi}\right)^{\frac{1}{2}} \int_{\Xi} e^{i k \mathcal{S}(\mathbf{x}, \xi)} A(\mathbf{x}, \xi) d \xi, \quad \xi \in \Xi \subset \mathbb{R}_{\xi}
$$

(see Kravtsov [KR], Ludwig [LU] and the excellent exposition by Duistermaat [DUI]).

Here $\mathcal{S}$ and $A$ satisfy the eikonal equation (2.3) and the transport equation (2.4), respectively, identically with respect to $\xi$. Such an integral can be regarded as a continuous superposition of oscillatory functions of the form (2.2). The physical motivation underlying the Kravtsov-Ludwig technique is the fact that in every small region in which the refraction index of the medium can be considered as constant and the wave front as plane, the field can be represented as a superposition of plane waves $A e^{i \mathcal{S}}$, where $A$ and $\nabla \mathcal{S}$ vary slowly in transition from one region to the next.

The integral (2.13) is computed for large $k$ applying the stationary phase lemma (see, e.g., $[\mathrm{BH}]$, p. 219) and this requires the local form of the phase function $\mathcal{S}=\mathcal{S}(\mathbf{x}, \xi)$ to be known. Local representation theorems for the phase function according to the type of caustic appearing are derived by the methods of singularity theory (see, e.g., [AVH], [GSC]). In the case of usual (single phase) geometrical optics we can take $\mathcal{S}(\mathbf{x}, \xi)=$ $\phi(\mathbf{x})-\xi^{2}$. In the simplest case of a smooth caustic (fold) the appropriate phase (see [GSC], [GS], p. 431, Proposition 6.1, and [KR]) has the form

$$
\mathcal{S}(\mathbf{x}, \xi)=\phi(\mathbf{x})+\xi \rho_{1}(\mathbf{x})-\frac{\xi^{3}}{3}
$$


while in the case of a cusp caustic it can be shown ([GS], p. 441, Proposition 7.1, [KR]) that the phase function must have the representation

$$
\mathcal{S}(\mathbf{x}, \xi)=\phi(\mathbf{x})+\rho_{1}(\mathbf{x}) \xi-\rho_{2}(\mathbf{x}) \frac{\xi^{2}}{2}+\frac{\xi^{4}}{4} .
$$

If there is more than one simple stationary point $\xi_{j}(\mathbf{x})$, that is, $\partial_{\xi}\left(\mathcal{S}\left(\mathbf{x}, \xi_{j}(\mathbf{x})\right)=0\right.$ and $\partial_{\xi}^{2} \mathcal{S}\left(\mathbf{x}, \xi_{j}(\mathbf{x})\right) \neq 0$, we obtain the asymptotic expansion

$$
u(\mathbf{x}) \sim \sum_{j} A_{0}^{j}(\mathbf{x}) e^{i k \mathcal{S}_{j}(\mathbf{x})}
$$

where

$$
\mathcal{S}_{j}(\mathbf{x})=\mathcal{S}\left(\mathbf{x}, \xi_{j}(\mathbf{x})\right)
$$

and

$$
A_{0}^{j}(\mathbf{x})=\exp \left(i\left(\frac{\pi}{2}+\operatorname{sgn}\left(\partial_{\xi}^{2} \mathcal{S}\left(\mathbf{x}, \xi_{j}(\mathbf{x})\right)\right)\right)\right) \frac{A\left(\mathbf{x}, \xi_{j}(\mathbf{x})\right)}{\sqrt{\left|\partial_{\xi}^{2} \mathcal{S}\left(\mathbf{x}, \xi_{j}(\mathbf{x})\right)\right|}} .
$$

The amplitudes (2.18) solve the zero-order transport equation (2.4), and (2.13) reduces asymptotically to $(2.2)$ with $l=0$. Note that $S_{j}$ coincides with the various branches of $S$ obtained by (2.10); so the branches of $S$ given by (2.10) have to be known in order to compute the wavefield by (2.16). The summation in (2.16) extends over all the stationary points.

However, the expansion (2.16) fails if the multiplicity of the stationary points is greater than one, i.e., $\partial_{\xi}^{2} \mathcal{S}\left(\mathbf{x}, \xi_{j}(\mathbf{x})\right)=0$ (obviously this can happen at certain points $\mathbf{x}$ for the phases (2.14), (2.15)), and the appearance of multiple stationary points is associated with the formation of caustics. In these cases, we can use (2.14) and (2.15) directly in the integral representation (2.13) together with stationary phase lemmas, to obtain the wavefield in a neighborhood of the caustic by matching properly with the geometrical optics field (see, e.g., [KKM], [HA], [HS]).

It becomes therefore evident that the asymptotic evaluation of the high-frequency wavefield in the region of caustics requires the study of the geometry of the multivalued solution.

3. Geometric solutions to the eikonal equation. In this section we study the multivalued solutions of the eikonal equation

$$
\left(\frac{\partial S}{\partial x}(x, z)\right)^{2}+\left(\frac{\partial S}{\partial z}(x, z)\right)^{2}-\eta^{2}(z)=0, \quad(x, z) \in \mathbb{R}^{2}
$$

as they are obtained by the method of characteristics (i.e., by means of Eq. (2.10)), in the geometric framework of Legendrian immersions, and we obtain classification results for singularities of the considered initial value problems.

We assume that the refraction index $\eta$ depends only on the variable $z$ (stratified medium) and that it is a continuous and piecewise smooth function, i.e., there exist finitely many numbers

$$
0=c_{0}>c_{1}>\cdots>c_{n}
$$


such that $\eta_{i}:\left(c_{i-1}, c_{i}\right) \rightarrow \mathbb{R}_{0}^{+}(i=1, \ldots, n)$ is a smooth function with $\eta_{i-1}\left(c_{i-1}\right)=\eta_{i}\left(c_{i}\right)$ where $\eta_{i}=\left.\eta\right|_{\left[c_{i-1}, c_{i}\right]}$. We will also consider the case where the solution is restricted on the half space with boundary at $z=0$ where the rays reflect on the boundary. We first study the problem when the solution is considered in the whole space and $\eta(z)$ is smooth. We then study the case where $\eta(z)$ is piecewise smooth and $\eta^{\prime}(z)$ is discontinuous across the points $c_{i}$. New phenomena arise in this case as the characteristics change velocity across the interface $z=c_{i}$, and they might separate and create shadow zones. We finally discuss the problem where the solution is considered in a half space, the boundary of which causes reflection of caustics and it leads to the formation of shadow zones inside the medium.

3.1. The geometric framework. We consider the Hamiltonian function $H: \mathbb{R}^{4} \rightarrow \mathbb{R}$ given by $H(x, z, p, q)=\frac{1}{2}\left(p^{2}+q^{2}-\eta^{2}(z)\right)$ where $(x, z) \in \mathbb{R}^{2}$. Even if there is no boundary, the situation is still complicated when the refraction index $\eta(z)$ is non-smooth. So, we deal first with the case of smooth $\eta(z)$.

We start by describing the geometric framework for the multivalued (geometric) solutions of the eikonal equation by the method of characteristics. For this, we consider the phase space $\mathbb{R}^{4}$ with coordinates $(x, z, p, q)$ equipped with the canonical twoform $\omega=d p \wedge d x+d q \wedge d z$, and the canonical projection $\pi: \mathbb{R}^{4} \rightarrow \mathbb{R}^{2}$ given by $\pi(x, z, p, q)=(x, z)$. The one-form $\alpha=p d x+q d z$, which is characterized by the relation $d \alpha=\omega$, is called the Liouville form.

We define an eikonal hypersurface as the hypersurface $\mathcal{H}=H^{-1}(0)$ in $\mathbb{R}^{4}$. This hypersurface has the following basic properties:

(1) $\mathcal{H}$ is a smooth 3 -dimensional submanifold in $\mathbb{R}^{4}$;

(2) $\mathcal{H}$ is transversal to the fibre $\pi^{-1}(x, z)$ for any $(x, z) \in \mathbb{R}^{2}$;

(3) the intersection $\mathcal{H} \cap \pi^{-1}(x, z)=\left\{(x, z, p, q) \mid p^{2}+q^{2}=\eta^{2}(z)\right\}$ is a circle in the fibre $\pi^{-1}(x, z)$ with radius $\eta(z)$;

(4) the restriction of the Liouville form $\alpha$ to $\mathcal{H}$ gives a contact structure on $\mathcal{H}$, i.e., $\alpha \mid \mathcal{H} \wedge d(\alpha \mid \mathcal{H}) \neq 0$.

The above properties are easily verified, since the eikonal hypersurface is the image of the embedding

$$
f: \mathbb{R}^{2} \times S^{1} \rightarrow \mathbb{R}^{4},
$$

given by $f\left(x, z, e^{i \theta}\right)=(x, z, \eta(z) \cos \theta, \eta(z) \sin \theta)$. Here, we give only the proof of (4). Employing the local representation

$$
f(x, z, \theta)=(x, z, \eta(z) \cos \theta, \eta(z) \sin \theta), \quad 0<\theta<\pi,
$$

and taking into account that $f^{*} \alpha=\eta(z) \cos \theta d x+\eta(z) \sin \theta d z$, we have

$$
d f^{*} \alpha=\eta^{\prime}(z) \cos \theta d z \wedge d x+\eta(z) \sin \theta d x \wedge d \theta+\eta(z) \cos \theta d \theta \wedge d z .
$$

Then, it follows that

$$
f^{*} \alpha \wedge d f^{*} \alpha=\eta^{2}(z) d x \wedge d \theta \wedge d z \neq 0,
$$

which means that $\alpha \mid \mathcal{H}$ gives a contact structure on $\mathcal{H}$.

The classical method of the characteristics has been formulated in contact geometry (see [LY]). In this case the characteristic vector field $X_{H}$ on the phase space $\mathbb{R}^{4}$ is given 
by

$$
X_{H}=p \frac{\partial}{\partial x}+q \frac{\partial}{\partial z}+\eta^{\prime}(z) \eta(z) \frac{\partial}{\partial q}
$$

and it corresponds to the following system of ordinary differential equations:

$$
\dot{x}=p, \quad \dot{z}=q, \quad \dot{p}=0, \quad \dot{q}=\eta^{\prime}(z) \eta(z) .
$$

The flow that generates the characteristic vector field is called the Hamiltonian flow (or bicharacteristic flow).

The characteristic vector field $X_{H}$ has the following well-known properties:

(1) $\alpha \circ X_{H}=\eta^{2}(z)$ on $\mathcal{H}$;

(2) $X_{H}$ is tangent to the eikonal hypersurface $\mathcal{H}$.

The property (1) follows from a direct calculation. The property (2) follows from the fact that the gradient

$$
\operatorname{grad} H=-\eta^{\prime}(z) \eta(z) \frac{\partial}{\partial z}+p \frac{\partial}{\partial p}+q \frac{\partial}{\partial q}
$$

of the vector field of $H$ satisfies $\left\langle X_{H}\right.$, $\left.\operatorname{grad} H\right\rangle=0$. This property means that all Hamiltonian flows starting from points in $\mathcal{H}$ are completely contained in $\mathcal{H}$.

The notion of multivalued solutions of $(E)$. We consider the coordinates $(x, z, y, p, q)$ of $\mathbb{R}^{5}$ and the canonical 1-form $\Theta=d y-p d x-q d z$. This form satisfies $\Theta \wedge d \Theta^{2} \neq 0$, i.e., it gives the canonical structure on $\mathbb{R}^{5}$.

We introduce also the extended eikonal hypersurface

$$
\widetilde{\mathcal{H}}=\left\{(x, z, y, p, q) \mid p^{2}+q^{2}-\eta^{2}(z)=0\right\},
$$

which clearly satisfies $\tilde{\mathcal{H}}=\mathcal{H} \times \mathbb{R}$.

For a classical solution $y=S(x, z)$ of $(E)$, we have the embeddings

$$
\begin{aligned}
& D S: \mathbb{R}^{2} \rightarrow \mathbb{R}^{4} ; \quad D S(x, z)=\left(x, z, \frac{\partial S}{\partial x}, \frac{\partial S}{\partial z}\right), \\
& j^{1} S: \mathbb{R}^{2} \rightarrow \mathbb{R}^{5} ; \quad j^{1} S(x, z)=\left(x, z, S, \frac{\partial S}{\partial x}, \frac{\partial S}{\partial z}\right) .
\end{aligned}
$$

Since $y=S(x, z)$ is a smooth function, we have

$$
\left(j^{1} S\right)^{*} \Theta=d S(x, z)-\frac{\partial S}{\partial x} d x-\frac{\partial S}{\partial z} d z=0
$$

and

$$
(D S)^{*} \omega=(D S)^{*}(d \alpha)=d\left(D S^{*} \alpha\right)=d\left(\frac{\partial S}{\partial x} d x+\frac{\partial S}{\partial z} d z\right)=d d S=0 .
$$

If we consider the projection $\Pi: \mathbb{R}^{5} \rightarrow \mathbb{R}^{4}$ given by $\Pi(x, z, y, p, q)=(x, z, p, q)$, we also have $\Pi \circ j^{1} S=D S$. Moreover, it is clear that $j^{1} S\left(\mathbb{R}^{2}\right) \subset \tilde{\mathcal{H}}$ and $D S\left(\mathbb{R}^{2}\right) \subset \mathcal{H}$.

Let $L$ be a 2-dimensional manifold and $i_{1}: L \rightarrow \mathbb{R}^{4}$ and $i_{2}: L \rightarrow \mathbb{R}^{5}$ be immersions. We say that $i_{1}$ is a Lagrangian immersion if $i_{1}^{*} \omega=0$ and $i_{2}$ is a Legendrian immersion if $i_{2}^{*} \Theta=0$. We call $i_{2}$ a Legendrian lift of $i_{1}$ if $\Pi \circ i_{2}=i_{1}$. For any Legendrian immersion $i_{2}: L \rightarrow \mathbb{R}^{5}$, we can easily show that $\Pi \circ i_{2}: L \rightarrow \mathbb{R}^{4}$ is a Lagrangian immersion. If $L$ is simply connected, it is well known that any Lagrangian immersion has a Legendrian 
lift (cf. Lychagin [LY]). Then, we introduce the notion of the multivalued solution as follows.

DEFINITION 3.1. A multivalued solution (or geometric solution) of (E) is a Legendrian immersion $i_{2}: L \rightarrow \mathbb{R}^{5}$ such that $i_{2}(L) \subset \tilde{\mathcal{H}}$.

The corresponding Lagrangian immersion $i_{1}=\Pi \circ i_{2}$ satisfies $i_{1}(L) \subset \mathcal{H}$, and we call it a Lagrangian solution of $(E)$. We call the set $W\left(i_{2}\right)=\tilde{\pi} \circ i_{2}(L)$ the wave front set of $i_{2}$, where $\tilde{\pi}: \mathbb{R}^{5} \rightarrow \mathbb{R}^{3}$ is the canonical projection given by $\tilde{\pi}(x, z, y, p, q)=(x, z, y)$. As in the case of the classical solution, $W\left(i_{2}\right)$ is the graph of the multivalued solution $i_{2}$.

For a classical solution $y=S(x, z)$, we have $\pi \circ D S(x, z)=(x, z)$ and $\tilde{\Pi} \circ j^{1} S(x, z)=$ $(x, z, S(x, z))$, so that the mappings $D S, j^{1} S$ are nonsingular. For a Lagrangian immersion $i_{1}: L \rightarrow \mathbb{R}^{4}$, the critical value set of $\pi \circ i_{1}$ is called the caustic of $i_{1}$, and it is denoted by $C_{i_{1}}$. If the coordinate representation of $i_{1}$ is given by $i_{1}\left(u_{1}, u_{2}\right)=$ $\left(x\left(u_{1}, u_{2}\right), z\left(u_{1}, u_{2}\right), p\left(u_{1}, u_{2}\right), q\left(u_{1}, u_{2}\right)\right)$, then we have

$$
C_{i_{1}}=\left\{\left(x\left(u_{1}, u_{2}\right), z\left(u_{1}, u_{2}\right)\right) \in \mathbb{R}^{2} \mid \operatorname{det} \frac{\partial(x, z)}{\partial\left(u_{1}, u_{2}\right)}=0\right\} .
$$

Since $i_{2}: L \rightarrow \mathbb{R}^{5}$ is a Legendrian lift of $i_{1}$, the caustics of $i_{1}$ are equal to the critical value set of $\pi \circ \Pi \circ i_{2}$ by definition. On the other hand, the locus of the singularities of the wave front set of $i_{2}$ is

$$
\mathcal{S}\left(W\left(i_{2}\right)\right)=\left\{\left(x\left(u_{1}, u_{2}\right), z\left(u_{1}, u_{2}\right), y\left(u_{1}, u_{2}\right) \mid \operatorname{rank} \frac{\partial(x, z, y)}{\partial\left(u_{1}, u_{2}\right)} \leq 1\right\}\right.
$$

where $i_{2}\left(u_{1}, u_{2}\right)=\left(x\left(u_{1}, u_{2}\right), z\left(u_{1}, u_{2}\right), y\left(u_{1}, u_{2}\right), p\left(u_{1}, u_{2}\right), q\left(u_{1}, u_{2}\right)\right)$. We can easily show that $i_{2}^{*} \Theta=0$ if and only if

$$
\begin{aligned}
& \frac{\partial y}{\partial u_{1}}=p\left(u_{1}, u_{2}\right) \frac{\partial x}{\partial u_{1}}+q\left(u_{1}, u_{2}\right) \frac{\partial z}{\partial u_{1}} \\
& \frac{\partial y}{\partial u_{2}}=p\left(u_{1}, u_{2}\right) \frac{\partial x}{\partial u_{2}}+q\left(u_{1}, u_{2}\right) \frac{\partial z}{\partial u_{2}}
\end{aligned}
$$

Therefore, we have

$$
\operatorname{rank} \frac{\partial(x, z, y)}{\partial\left(u_{1}, u_{2}\right)}=\operatorname{rank} \frac{\partial(x, z)}{\partial\left(u_{1}, u_{2}\right)}
$$

which means that

$$
C_{i_{1}}=\left\{\left(x\left(u_{1}, u_{2}\right), z\left(u_{1}, u_{2}\right)\right) \mid\left(x\left(u_{1}, u_{2}\right), z\left(u_{1}, u_{2}\right), S\left(u_{1}, u_{2}\right)\right) \in \mathcal{S}\left(W\left(i_{2}\right)\right)\right\} .
$$

Multivalued solutions of initial value problem for $(E)$. In order to introduce this notion of solution, we need some further definitions which are necessary for the study of the geometric solution that now goes through an initial manifold. Let $\ell: I \rightarrow \mathcal{H}$ be a regular curve of the form $\ell(\theta)=(x(\theta), z(\theta), p(\theta), q(\theta))$ with $p(\theta) x^{\prime}(\theta)+q(\theta) z^{\prime}(\theta)=0$, where $I$ is an open interval. Here, $\ell$ is regular if $\ell^{\prime}(\theta) \neq 0$ for any $\theta \in I$. It follows that

$$
\ell^{*} \alpha=p(\theta) d x(\theta)+q(\theta) d z(\theta)=\left(p(\theta) x^{\prime}(\theta)+q(\theta) z^{\prime}(\theta)\right) d \theta=0 .
$$

This condition is equivalent to the fact that $\ell$ is a Legendrian curve in the contact manifold $\mathcal{H}$. The Hamiltonian vector field along $\ell$ is $X_{H} \circ \ell(\theta)=\left(p(\theta), q(\theta), 0, \eta(z(\theta)) \eta^{\prime}(z(\theta))\right)$ and the tangent vector of $\ell$ is $\ell^{\prime}(\theta)=\left(x^{\prime}(\theta), z^{\prime}(\theta), p^{\prime}(\theta), q^{\prime}(\theta)\right)$. If there exist $\theta_{0} \in I$ and a real number $\lambda$ such that $\ell^{\prime}\left(\theta_{0}\right)=\lambda X_{H} \circ \ell\left(\theta_{0}\right)$, then $0=\ell^{*} \alpha\left(\theta_{0}\right)=\alpha\left(\ell^{\prime}\left(\theta_{0}\right)\right)=\lambda \alpha \circ X_{H} \circ \ell\left(\theta_{0}\right)=$ 
$\lambda \eta(z(\theta))^{2} \neq 0$, a contradiction. Therefore, the vectors $X_{H} \circ \ell(\theta), \ell^{\prime}(\theta)$ always have different direction, and the noncharacteristic condition is automatically satisfied for any Legendrian curve $\ell: I \rightarrow \mathcal{H}$.

Definition 3.2. We say that a geometric Cauchy problem (GCP) is given for (E) if a Legendrian curve $\ell: I \rightarrow \mathcal{H}$ is given, and we look for a geometric solution $L$ of $(\mathrm{E})$ such that $\ell(I) \subset i_{1}(L)$.

In order to solve the GCP, we consider the initial value problem for the characteristic equations

$$
\begin{cases}\dot{x}=p, \quad \dot{z}=q, & \dot{p}=0, \quad \dot{q}=\eta(z) \eta^{\prime}(z), \\ x(0, \theta)=x(\theta), & z(0, \theta)=z(\theta), \quad p(0, \theta)=p(\theta), \quad q(0, \theta)=q(\theta) .\end{cases}
$$

The solution of $(\mathrm{C})$ has the form

$$
\{x(\tau, \theta)=p(\theta) \tau+x(\theta), \quad z(\tau, \theta), \quad p(\tau, \theta)=p(\theta), \quad q(\tau, \theta)=\dot{z}(\tau, \theta)\},
$$

where

$$
\ddot{z}(\tau, \theta)=\eta(z(\tau, \theta)) \eta^{\prime}(z(\tau, \theta)), \quad z(0, \theta)=z(\theta), \quad \text { and } \quad \dot{z}(0, \theta)=q(\theta) .
$$

For the smooth map $\Gamma: \mathbb{R}_{+} \times I \rightarrow \mathbb{R}^{4}$ defined by

$$
\Gamma(\tau, \theta)=(p(\theta) \tau+x(\theta), z(\tau, \theta), p(\theta), \dot{z}(\tau, \theta)),
$$

we have

$$
\Gamma^{*} \omega=\left(p^{\prime}(\theta) p(\theta)+\frac{\partial \dot{z}}{\partial \theta}(\tau, \theta) \dot{z}(\tau, \theta)-\ddot{z}(\tau, \theta) \frac{\partial z}{\partial \theta}(\tau, \theta)\right) d \theta \wedge d \tau .
$$

The Hamiltonian flows are completely contained in the eikonal hypersurface, and the image of $\Gamma$ is included in $\mathcal{H}$. Differentiating the relation $p(\theta)^{2}+\dot{z}(\tau, \theta)^{2}-\eta^{2}(z(\tau, \theta)) \equiv 0$ with respect to $\theta$, and substituting in (3.16), we obtain $\Gamma^{*} \omega=0$. Since Hamiltonian flows do not cross on $\mathbb{R}^{4}$ and the initial submanifold satisfies the noncharacteristic condition, $\Gamma$ is a regular surface, and the rank of the Jacobi matrix

$$
\left(\begin{array}{cccc}
p(\theta) & \dot{z}(\tau, \theta) & 0 & \ddot{z}(\tau, \theta) \\
p^{\prime}(\theta) \tau+x^{\prime}(\theta) & \frac{\partial z}{\partial \theta}(\tau, \theta) & p^{\prime}(\theta) & \frac{\partial \dot{z}(\tau, \theta)}{\partial \theta}
\end{array}\right)
$$

is always equal to 2 . Therefore, $\Gamma$ is a Lagrangian immersion and Image $\Gamma \subset \mathcal{H}$. We also have

$$
\Gamma^{*}(\alpha)=p(\theta)\left(p^{\prime}(\theta) \tau+p(\theta) x^{\prime}(\theta)+\dot{z} \frac{\partial z}{\partial \theta}(\tau, \theta)\right) d \theta+\eta(z(\tau, \theta)) \eta^{\prime}(z(\tau, \theta)) d \tau
$$

Since $d \alpha=\omega$, we have $d \Gamma^{*} \alpha=\Gamma^{*} d \alpha=\Gamma^{*} \omega=0$. By the Poincaré Lemma, there exists (at least locally) a function $S(\tau, \theta)$ such that $d S=\Gamma^{*} \alpha$. Moreover, since $\mathbb{R}^{+} \times I$ is simply connected, we can construct the function $S(\tau, \theta)$ globally. In this case we have

$$
d S\left(\left.\frac{\partial}{\partial \tau}\right|_{(\tau, \theta)}\right)=\eta(z(\tau, \theta))
$$

and

$$
d S\left(\left.\frac{\partial}{\partial \theta}\right|_{(\tau, \theta)}\right)=p(\theta)\left(p^{\prime}(\theta) \tau+p(\theta) x^{\prime}(\theta)+\dot{z} \frac{\partial z}{\partial \theta}(\tau, \theta)\right)
$$


We now define a smooth map $\widetilde{\Gamma}: \mathbb{R}^{+} \times I \rightarrow \mathbb{R}^{5}$

$$
\widetilde{\Gamma}(\tau, \theta)=(p(\theta) \tau+x(\theta), z(\tau, \theta), S(\tau, \theta), \dot{z}(\tau, \theta)),
$$

which since $d S=\Gamma^{*} \alpha, \widetilde{\Gamma}$, is the Legendrian lift of $\Gamma$. Eventually, we will construct the Lagrangian solution $\Gamma$ and the Legendrian solution $\widetilde{\Gamma}$ for the initial data $\ell: I \rightarrow \mathcal{H}$ for the eikonal equation $(\mathrm{E})$.

The curve $\pi \circ \ell: I \rightarrow \mathbb{R}^{2}$ is called the initial front of $G C P$ and the curve $\left.\pi \circ \Gamma\right|_{S(\tau, \theta)=s_{0}}$ : $I \rightarrow \mathbb{R}^{2}$, denoted by $\tilde{\ell}_{s_{0}}$, is called the $s_{0}$-front of the Legendrian solution. In this case, the wave front set $W(\widetilde{\Gamma})$ is called a big front. We also call the curve $\left.\pi \circ \Gamma\right|_{\theta=\theta_{0}}: \mathbb{R}^{+} \rightarrow \mathbb{R}^{2}$ a ray for any fixed $\theta_{0} \in I$ and we denote it by $R_{\theta_{0}}(\tau)$.

3.2. Classification of singularities for smooth Hamiltonians. In order to better understand the geometric characteristics of the multivalued solutions, we will first study some examples corresponding to distinctive initial data usually arising in applications.

(a) The point source case: For any fixed $z_{0} \in \mathbb{R}$, we consider that the rays are starting from $\left(0, z_{0}\right)$ (see $[\mathrm{AK}],[\mathrm{B}]$ for the high-frequency and ray-theoretic treatment of a point source). We consider the following initial curve

$$
\ell(\theta)=\left(0, z_{0}, \eta\left(z_{0}\right) \cos \theta, \eta\left(z_{0}\right) \sin \theta\right), \quad \theta \in\left(-\frac{\pi}{2}, \frac{\pi}{2}\right)
$$

in $\mathcal{H}$. It is easy to show that $\ell$ is regular and isotropic. The Lagrangian solution is given by

$$
\Gamma(\tau, \theta)-\left(\tau \eta\left(z_{0}\right) \cos \theta, z(\tau, \theta), \eta\left(z_{0}\right) \cos \theta, \dot{z}(\tau, \theta)\right),
$$

where $z(0, \theta)=z_{0}, \dot{z}(0, \theta)=\eta\left(z_{0}\right) \sin \theta$ and $\ddot{z}(\tau, \theta)=\eta(z(\tau, \theta)) \eta^{\prime}(z(\tau, \theta))$.

(b) The smooth initial front case: Let $\gamma: I \rightarrow \mathbb{R}^{2}$ be a smooth regular curve given by $\gamma(\theta)=(x(\theta), z(\theta))$. We assume that the rays are starting from the image of $\gamma$ and that their direction is perpendicular to $\gamma(\theta)$. Consider the following initial curve:

$$
\ell_{\gamma}(\theta)=\left(x(\theta), z(\theta), \frac{-\eta(z(\theta))}{\sqrt{x^{\prime}(\theta)^{2}+z^{\prime}(\theta)^{2}}} z^{\prime}(\theta), \frac{\eta(z(\theta))}{\sqrt{x^{\prime}(\theta)^{2}+z^{\prime}(\theta)^{2}}} x^{\prime}(\theta)\right)
$$

in $\mathcal{H}$. In this case the Lagrangian solution is

$$
\Gamma(\tau, \theta)=\left(\frac{-\eta(z(\theta))}{\sqrt{x^{\prime}(\theta)^{2}+z^{\prime}(\theta)^{2}}} z^{\prime}(\theta) \tau+x(\theta), z(\tau, \theta), \frac{-\eta(z(\theta))}{\sqrt{x^{\prime}(\theta)^{2}+z^{\prime}(\theta)^{2}}} z^{\prime}(\theta), \dot{z}(\tau, \theta)\right),
$$

where

$$
z(0, \theta)=z(\theta), \quad \dot{z}(0, \theta)=\frac{\eta(z(\theta))}{\sqrt{x^{\prime}(\theta)^{2}+z^{\prime}(\theta)^{2}}} x^{\prime}(\theta)
$$

and

$$
\ddot{z}(\tau, \theta)=\eta(z(\tau, \theta)) \eta^{\prime}(z(\tau, \theta)) .
$$

In case $(\mathrm{a})$ the Jacobian of $(\tau, \theta) \rightarrow(x, z)$ is given by

$$
J(\tau, \theta)=\eta\left(z_{0}\right)\left\{\cos \theta \frac{\partial z}{\partial \theta}(\tau, \theta)+\tau \dot{z}(\tau, \theta) \sin \theta\right\} .
$$


Since $\frac{\partial z}{\partial \theta}(0, \theta)=0$, we have $J(0, \theta)=0$. Moreover, we have $\frac{\partial \dot{z}(0, \theta)}{\partial \theta}=\eta\left(z_{0}\right) \cos \theta>0$, so that $\frac{\partial \dot{z}(\tau, \theta)}{\partial \theta}>0$ for sufficiently small $\tau>0$. It follows that $\frac{\partial z}{\partial \theta}(\tau, \theta)>0$ for sufficiently small $\tau>0$. Since $\dot{z}(0, \theta) \sin \theta=\eta\left(z_{0}\right) \sin ^{2} \theta>0$ for any $\theta \in\left(-\frac{\pi}{2}, \frac{\pi}{2}\right) \backslash\{0\}, \tau \dot{z}(\tau, \theta) \sin \theta>$ 0 for sufficiently small $\tau>0$. Therefore, $J(\tau, \theta)>0$ for sufficiently small $\tau>0$ and any $\theta \in\left(-\frac{\pi}{2}, \frac{\pi}{2}\right)$. This means that the source point $\left(0, z_{0}\right)$ is an isolated caustic. Moreover, the $\varepsilon$-front $\pi \circ \Gamma_{\varepsilon}$ for sufficiently small $\varepsilon>0$ is a regular curve in $\mathbb{R}^{2}$. It we apply the characteristic method at the $\varepsilon$-front, i.e., $\pi \circ \Gamma_{\varepsilon}$ is the smooth initial front, the solution we obtain agrees with the one starting from the source point. The same conclusions apply to case b) since $J(0, \theta)=-\eta(z(\theta)) \sqrt{x^{\prime}(\theta)^{2}+z^{\prime}(\theta)^{2}}<0$.

Let us now compute the Lagrangian solution and the caustics for some simple refraction indices.

EXAMPLE 1 (Point source in a linear layer, $[K R]$ ). We consider the case of a point source located at the point $\left(x=0, z=z_{0}\right)$ in a medium with linear refraction index

$$
\eta^{2}(z)=1-z / d, \quad z \leq d .
$$

We set $\eta_{0}=\sqrt{1-z_{0} / d}$. The Lagrangian solution is

$$
\Gamma(\tau, \theta)=\left(\eta_{0} \tau \cos \theta, \quad-\frac{1}{4 d} \tau^{2}+\eta_{0} \tau \sin \theta+z_{0}, \quad \eta_{0} \cos \theta, \quad-\frac{\tau}{2 d}+\eta_{0} \sin \theta\right) .
$$

The Jacobian of $(x(\tau, \theta), z(\tau, \theta))$ is given by

$$
J(\tau, \theta)=\eta_{0} \tau\left(1-\eta_{0} \tau \frac{\sin \theta}{2 d \eta_{0}^{2}}\right) .
$$

The caustic consists of the isolated point $\left(0, z_{0}\right)$ and the parabola

$$
z=d \eta_{0}^{2}\left(1-\frac{x^{2}}{4 d \eta_{0}^{2}}\right)+z_{0}
$$

which is a fold singularity.

EXAMPLE 2 (Evolution of a parabolic initial front, [V1]). We look for the solution of

$$
(\nabla S)^{2}=1,\left.\quad S\right|_{\gamma}=0,\left.\quad \frac{\partial S}{\partial \nu}\right|_{\gamma}=1
$$

where $\gamma:\left\{z^{2}=2 a x, a>0\right\}$ is the initial front, and $\nu$ is the normal to $\gamma$ directed towards the positive $x$-axis. If we consider the parametric representation of $\gamma$ in the form

$$
\left\{z=\theta, \quad x=\frac{\theta^{2}}{2 a}, \quad-\infty<\theta<+\infty\right\}
$$

the Lagrangian solution is

$$
\Gamma(\tau, \theta)=\left(\frac{a}{\sqrt{a^{2}+\theta^{2}}} \tau+\frac{\theta^{2}}{2 a}, \quad-\frac{\theta}{\sqrt{a^{2}+\theta^{2}}} \tau+\theta, \quad \frac{a}{\sqrt{a^{2}+\theta^{2}}}, \quad-\frac{\theta}{\sqrt{a^{2}+\theta^{2}}}\right) .
$$

The Jacobian of the mapping $(\tau, \theta) \rightarrow(x, z)$ is

$$
J(\tau, \theta)=\frac{1}{a} \sqrt{a^{2}+\theta^{2}}-\frac{a}{a^{2}+\theta^{2}} \tau,
$$

and the caustic is a cusp singularity given by

$$
x=a+\frac{3 a^{1 / 3}}{2} z^{2 / 3} .
$$


EXAMPLE 3 (Point source in parabolic waveguide, $[\mathrm{BR}]$ ). We consider the ray field of a source at $z_{0}=0$ in a medium with

$$
\eta(z)=1-a^{2} z^{2}
$$

in the strip bounded by the lines $z= \pm 1 / a$. The rays are given in parametric form by

$$
x(\tau, \theta)=\tau \cos \theta, \quad z(\tau, \theta)=\frac{1}{a} \sin \theta \sin (a \tau) .
$$

The Jacobian of the rays (3.38) vanishes at the points $(\tau, \theta)$ satisfying

$$
\tan (a \tau)=-a \tau \tan ^{2} \theta
$$

For $\theta \in(-\pi / 2, \pi / 2)$, it can easily be seen in a graphical way that (3.39) has an infinite sequence of roots $\tau_{c}=\tau_{c}(\theta)$, one in each interval $( \pm(2 m-1) \pi / 2, \pm m \pi), m=1,2, \ldots$, corresponding to the branches of the tangent function. So, the parametric equations for the caustic are

$$
a x_{c}(\theta)=a \tau_{c}(\theta) \cos \theta, \quad a z_{c}(\theta)=\sin \theta \sin \left(a \tau_{c}(\theta)\right)
$$

and this caustic has an infinity of branches.

Differentiating (3.39) and (3.40) with respect to $\theta$, we have

$$
d x_{c} / d \theta=d \tau_{c} / d \theta-\tau_{c} \sin \theta, \quad a d z_{c} / d \theta=\cos \theta \sin \left(a \tau_{c}\right)+a \sin \theta \cos \left(a \tau_{c}\right) d \tau_{c} / d \theta,
$$

where

$$
d \tau_{c} / d \theta=-\frac{2 a \tau_{c}(\theta) \tan \theta\left(1+\tan ^{2} \theta\right)}{1+a \tan ^{2} \theta+a^{2} \tau_{c}^{2}(\theta) \tan ^{4} \theta} .
$$

The cusp points of the caustic must satisfy

$$
d x_{c} / d \theta=d z_{c} / d \theta=0 .
$$

For any $\theta \neq 0,(3.41 b)$ and (3.42a) imply that $\tau_{c}^{2}<0$. For $\theta=0$, it follows that $\tau_{c}=m \pi / a, m=1,2, \ldots$, and the corresponding second and third derivatives are

$$
\begin{aligned}
& d^{2} x_{c} / d \theta^{2}=-3 m \pi / a \neq 0, \quad d^{2} z_{c} / d \theta^{2}=0, \\
& d^{3} z_{c} / d \theta^{3}=-6 m \pi \neq 0, \quad m=1,2, \ldots
\end{aligned}
$$

Therefore the points $\left(x_{c}=m \pi / a, z_{c}=0\right), m=1,2 \ldots$, are the cusp points of the caustic. Note that for $m=0$, the corresponding point of the caustic coincides with the source. Finally, it is easily seen that for $\theta= \pm(m-1 / 2) \pi$, all the branches of the caustic converge to the points $(x=0, z= \pm 1 /(2 a))$, and the whole caustic is confined between the lines $z= \pm 1 /(2 a)$ (see Fig. 1).

The above simple examples suggest that the common type of appearing singularities are either folds or cusps. As follows from Theorems 3.1, 3.2 and 3.3 below, these singularities are generic, in the sense that, for a given Hamiltonian, they appear for "almost all" initial data. So the scope of this section is to present various results on classification of the caustics $C_{\Gamma}$ and bifurcations of $\tau$-fronts $\pi \circ \Gamma_{\tau}(\theta)$ as the parameter $\tau$ increases, for generic initial data $\ell: I \rightarrow \mathcal{H}$ corresponding to the point source case or the smooth initial front case. 


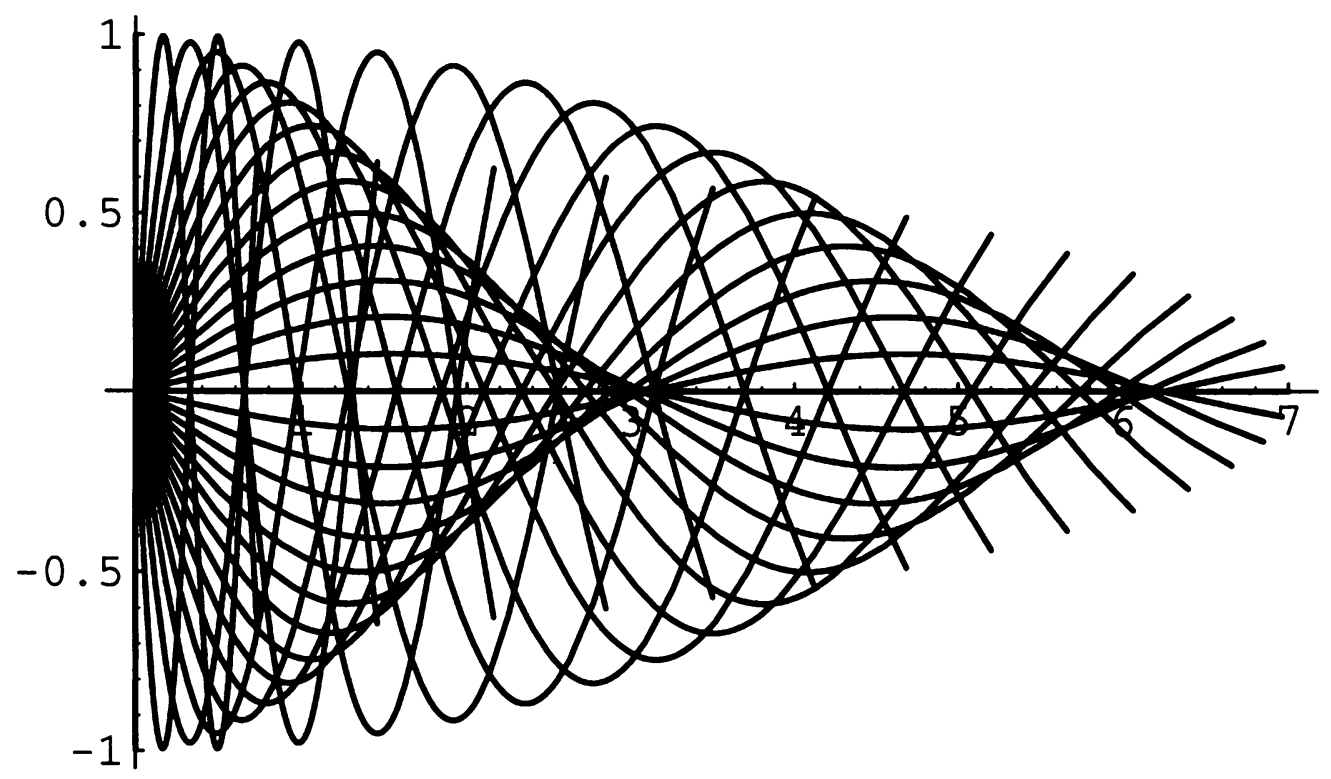

FIG. 1. Rays of a point source in a parabolic layer

For this we introduce the notion of graphlike Legendrian unfoldings and review some fundamental properties. Since we only consider local classifications, we describe those as germs. Let $\Lambda:\left(I \times J ;\left(\tau_{0}, \theta_{0}\right)\right) \rightarrow \mathbb{R}^{5}$ be a Legendrian immersion germ, where $I, J$ are open intervals.. We say that $\Lambda(\tau, \theta)=(x(\tau, \theta), z(\tau, \theta), y(\tau, \theta), p(\tau, \theta), q(\tau, \theta))$ is a Legendrian unfolding if $\frac{\partial y}{\partial \tau}\left(\tau_{0}, \theta_{0}\right) \neq 0$. We have shown that $\frac{\partial S}{\partial t}\left(t_{0}, \theta_{0}\right)=\eta\left(\tau_{0}, \theta_{0}\right) \neq 0$. This means that the Legendrian solution $\widetilde{\Gamma}: \mathbb{R}_{+} \times I \rightarrow \mathbb{R}^{5}$ of the GCP with initial data $\ell: I \rightarrow \mathcal{H}$, is a Legendrian unfolding around any point $\left(\tau_{0}, \theta_{0}\right) \in \mathbb{R}_{+} \times I$.

We say that two Legendrian unfoldings $\Lambda_{i}:\left(I \times J,\left(\tau_{i}, \theta_{i}\right)\right) \rightarrow \mathbb{R}^{5}(i=0,1)$ are $P$-Legendrian equivalent if there exists a diffeomorphism germ

$$
\Phi:\left(\mathbb{R}^{5}, \Lambda_{0}\left(\tau_{0}, \theta_{0}\right)\right) \rightarrow\left(\mathbb{R}^{5}, \Lambda_{1}\left(\tau_{1}, \theta_{1}\right)\right)
$$

of the form

$$
\Phi(x, z, y, p, q)=\left(\phi_{1}(x, z, y), \phi_{2}(x, z, y), \phi_{3}(y), \phi_{4}(x, z, y, p, q), \phi_{5}(x, z, y, p, q)\right)
$$

such that $\Phi \circ \Lambda_{0}=\Lambda_{1}$, where $\Phi$ satisfies $\Phi^{*} \Theta=\lambda \Theta$ for some germ $\lambda(x, y, z, p, q)$ (i.e., $\Phi$ is a contact diffeomorphism germ). If $\Lambda_{0}$ and $\Lambda_{1}$ are $P$-Legendrian equivalent, then $\phi \circ \tilde{\pi} \circ$ $\Lambda_{0}=\tilde{\pi} \circ \Lambda_{1}$, where $\phi(x, y, z)=\left(\phi_{1}(x, y, z), \phi_{2}(x, y, z), \phi_{3}(x, y, z)\right)$. Since $\frac{\partial y}{\partial \tau}\left(\tau_{0}, \theta_{0}\right) \neq 0$, we may assume that $y(\tau, \theta)=\tau$ by a local change of the parameter $(\tau, \theta)$. It follows that this equivalence relation preserves bifurcation of wave fronts.

Moreover, we define the Lagrangian equivalence that preserves caustics. We say that two Legendrian unfoldings $\Lambda_{0}, \Lambda_{1}$ are Lagrangian equivalent if there exists a diffeomorphism germ

$$
\Phi:\left(\mathbb{R}^{4}, \pi \circ \Lambda_{0}\left(\tau_{0}, \theta_{0}\right)\right) \rightarrow\left(\mathbb{R}^{4}, \pi \circ \Lambda_{1}\left(\tau_{1}, \theta_{1}\right)\right)
$$


of the form

$$
\Phi(x, z, p, q)=\left(\phi_{1}(x, z), \phi_{2}(x, z), \phi_{3}(x, z, p, q), \phi_{4}(x, z, p, q)\right)
$$

such that $\Phi \circ \pi \circ \Lambda_{0}=\Lambda_{1}$ where $\Phi$ satisfies $\Phi^{*} \Theta=\Theta$ (i.e., $\Phi$ is a symplectic diffeomorphism germ).

By the general theory of Lagrangian singularities and graphlike Legendrian unfoldings (cf. $[\mathrm{AVH}],[\mathrm{IZ}]$ ), we have the following classification of graphlike unfoldings by the above two equivalence relations.

Theorem 3.1. (1) The "generic" Legendrian unfolding $\Lambda$ is $P$-Legendrian equivalent to the one of germs at the origin in the following list:

${ }^{0} A_{1}:(\tau, \theta, \tau, 1,0)$

${ }^{0} A_{2}:\left(\tau+2 \theta^{3},-3 \theta^{2}, \tau, 1, \theta\right)$

${ }^{1} A_{3}^{ \pm}:\left(\tau, 4 \theta^{3}+2 \theta \tau \pm\left(3 \theta^{4}+\theta^{2} \tau\right)+\tau, 1 \mp \theta^{2}, \pm \theta\right)$.

(2) The "generic" Legendrian unfolding $\Lambda$ is Lagrangian equivalent to the one of germs at the origin in the following list:

$A_{1}:(\tau, \theta, \tau, 1,0)$

$A_{2}:\left(\tau, \theta^{2}, 0, \theta\right)$

$A_{3}:\left(\tau, \pm \theta^{3}+\theta \tau, \frac{1}{2} \theta^{2}, \theta\right)$.

The pictures of bifurcations of fronts of ${ }^{0} A_{1},{ }^{0} A_{2},{ }^{1} A_{3}^{ \pm}$, of big fronts for ${ }^{0} A_{1},{ }^{0} A_{2},{ }^{1} A_{3}^{ \pm}$ and of caustics $A_{i}(i=2,3)$ are given in Chapter 5 of [AN]. However, the meaning of the "genericity" in Theorem 3.1 is too strong. It corresponds to the perturbation under all initial data and all Hamiltonian functions. For the scope of this paper we have to fix the Hamiltonian function. In this case the theorem of Bogaevskir [BO] still applies. Let $L(I, \mathcal{H})$ be the space of proper Legendrian immersions equipped with Whitney $C^{r}$ topology. Bogaevskiil's theorem can be interpreted as follows.

Theorem 3.2. There exists an open dense subset $O \subset L(I, \mathcal{H})$ such that for any $\ell \in$ $O$ the germ of the Lagrangian solution $\widetilde{\Gamma}(\tau, \theta)$ at any point $\left(\tau_{0}, \theta_{0}\right)$ is $P$-Legendrian equivalent to one of the germs ${ }^{0} A_{1},{ }^{0} A_{2},{ }^{1} A_{3}^{ \pm}$. Moreover, $\widetilde{\Gamma}(\tau, \theta)$ is Lagrangian equivalent to one of the germs $A_{r}, r=1,2,3$.

This theorem asserts that multivalued solutions of $(\mathrm{E})$ with generic initial data follow the exhaustive list of bifurcations of wavefronts and caustics. Moreover, we can apply the results given in [IZ] to obtain the following theorem.

ThEOREM 3.3. Let $\Lambda:\left(\mathbb{R}^{2}, 0\right) \rightarrow \mathbb{R}^{5}$ be Legendrian equivalent to one of the germs ${ }^{0} A_{1},{ }^{0} A_{2},{ }^{1} A_{3}^{ \pm}$. Then, there exists a Legendrian immersion $\ell:\left(I, \theta_{0}\right) \rightarrow \mathcal{H}$ such that the germ of the multivalued solution $\widetilde{\Gamma}$ of GCP for $(\mathrm{E})$ at $\left(\tau_{0}, \theta_{0}\right)$ is $P$-Legendrian equivalent to $\Lambda$.

3.3. Singularities for non-smooth Hamiltonians and domains with boundary. Before studying the proper geometric framework, we will present two examples where $\eta^{\prime}(z)$ is discontinuous. These examples give us an idea of how an interface affects the geometry of the rays and the formation of caustics depending on the position of the point source. 
EXAMPLE 4 (Mixed linear-homogeneous profile, [KO2], Sec. 3.3). The refraction index is given by

$$
\eta^{2}(z)= \begin{cases}1-2 a z, & 0<z<\frac{1}{2 a} \\ 1, & z<0\end{cases}
$$

a) Point source at the interface $\left(z_{0}=0\right)$.

Proceeding as in Example 1 we find that the caustic is given by

$$
z=\frac{1}{2 a}\left(1-a^{2} x^{2}\right), \quad z \geq 0 .
$$

If we only consider the part of the field for $x \geq 0$ (since the rest is symmetric with respect to the $z$-axis), the caustic for $z \geq 0$ is formed by the rays emitted with $\theta>\frac{\pi}{4}$. Rays emitted with $0 \leq \theta \leq \frac{\pi}{4}$ do not touch the caustic in $z>0$. All rays hit the $x$-axis at $x\left(\tau_{0}, \theta\right)=\frac{1}{\theta} \sin 2 \theta$ where $\tau_{0}(\theta)=\frac{2}{a} \sin \theta$, and they are refracted downwards in $z<0$ as straight lines with parametric equations

$$
x(\tau)=\tau \cos \theta+\frac{1}{a} \sin 2 \theta, \quad z(\tau)=-\tau \sin \theta .
$$

Using (2.11) and (3.49), we find that the caustic for $z<0$ is given in parametric form

$$
x(\theta)=\frac{1}{a}(1+\cos 2 \theta) \sin 2 \theta, \quad z(\theta)=-\frac{1}{a}(1-\cos 2 \theta) \cos 2 \theta,
$$

or, in cartesian coordinates, by

$$
16 a^{2} x^{2}=\left(27-18 \rho^{2}-\rho^{4}\right) \pm 8 \rho^{2}, \quad \rho=\sqrt{1+4 a z}, \quad-\frac{1}{4} \leq z \leq 0 .
$$

The caustic for $z<0$ exhibits a cusp at the point $A\left(\frac{3 \sqrt{3}}{4 a},-\frac{1}{4 a}\right)$ with slope $\left.\frac{d z}{d x}\right|_{A}=-\sqrt{3}$.

b) Point source in the homogeneous half space $\left(z_{0}<0\right)$.

The rays coming out from the source are straight lines for $z \leq 0$. The rays that refracted upwards in the layer $0<z<\frac{1}{2 a}$ are given in parametric form by

$$
x(\tau)=\tau \cos \theta-z_{0} \cot \theta, \quad z(\tau)=-\frac{a}{2} \tau^{2}+\tau \sin \theta
$$

and they form a caustic given by

$$
x(\theta)=\tau_{c}(\theta) \cos \theta-z_{0} \cot \theta, \quad z(\theta)=-\frac{a}{2} \tau_{c}^{2}(\theta)+\tau_{c}(\theta) \sin \theta, \quad 0<\theta \leq \pi / 2,
$$

where

$$
\tau_{c}=\left[a z_{0}+\sin ^{2} \theta+\left(\left(a z_{0}+\sin ^{2} \theta\right)^{2}-4 a z_{0} \sin ^{4} \theta\right)^{1 / 2}\right] /\left(2 a \sin ^{3} \theta\right) .
$$

The rays in the linear layer hit again the interface $z=0$, and they are refracted downwards into the homogeneous space. The rays refracted into $z \leq 0$ are given in parametric form by

$$
x(\tau)=\tau \cos \theta+x_{0}(\theta), \quad z(\tau)=-\tau \sin \theta
$$

where

$$
x_{0}(\theta)=\left(\frac{2}{a} \sin \theta-z_{0} \frac{1}{\sin \theta}\right) \cos \theta, \quad 0<\theta \leq \pi / 2
$$


and they form a caustic which is obtained from (3.53a) for

$$
\tau=\tau_{c}=\frac{1}{a}\left(2 \cos 2 \theta+\frac{a z_{0}}{\sin ^{2} \theta_{0}}\right) \sin \theta \geq 0 .
$$

From (3.54) it follows that for $\tau_{c} \geq 0$, the caustic consists of two branches (defined as a function of $x$ ) if $a z_{0}>-\frac{1}{4}$, while there is no caustic for $z<0$ if $a z_{0}<\frac{1}{4}$.

c) Point source inside the linear layer $\left(0<z_{0}<1 /(2 a)\right)$.

As in the first case the rays form a caustic, which is part of the parabola $z=\frac{1}{2 a}(1-$ $\left.a^{2} x^{2}\right)$ inside the layer $0<z<\frac{1}{2 a}$. The rays hit the interface $z=0$ at time $\tau_{0}(\theta)$ given by

$$
\tau_{0}(\theta)=\frac{\sin \theta+\sqrt{\sin ^{2} \theta+2 a z_{0}}}{a} .
$$

The downwards refracted rays are given in parametric form by

$$
x(\tau)=\tau \cos \theta+x_{0}(\theta), \quad z(\tau)=-\tau \sqrt{\sin ^{2} \theta+2 a z_{0}},
$$

where $x_{0}(\theta)=\tau_{0}(\theta) \cos \theta$. The caustic in $z<0$ is given by (3.56) for

$$
\begin{aligned}
\tau=\tau_{c}(\theta)=\frac{1}{a\left(1+2 a z_{0}\right)}( & \cos 2 \theta\left(\sin ^{2} \theta+2 a z_{0}\right) \\
& \left.+\sin \theta\left(2\left(1-a z_{0}\right)-3 \sin ^{2} \theta\right) \sqrt{\sin ^{2} \theta+2 a z_{0}}\right) .
\end{aligned}
$$

ExAMPle 5 (Point source in a parabolic layer [KO2]). The refraction index is given by

$$
\eta^{2}(z)= \begin{cases}1, & |z| \geq 1 \\ \left(1-a^{2}\right)+a^{2} z^{2}, & |z| \leq 1\end{cases}
$$

with $0<a<1$. We assume that the source is located at $\left(0, z_{0}\right)$ with $-1<z_{0}<0$. The rays are given by the parametric equations

$$
x(\tau)=\tau \cos \theta, \quad z(\tau)=z_{0} \cosh (a \tau)+\frac{\sin \theta}{a} \sinh (a \tau)
$$

There exists a critical angle $\theta=\theta_{\mathrm{cr}}=\arcsin \left(-a z_{0}\right)$ such that the corresponding ray tends asymptotically to the $x$-axis. Note also that the rays with $\theta>\theta_{\mathrm{cr}}$ have an inflection point on the axis $z=0$. The caustic points are obtained from the parametric equations of the rays for arrival times that satisfy

$$
\cos ^{2} \theta \sinh (a \tau)+a^{2} z_{0} \tau \sin \theta \sinh (a \tau)+a t \sin \theta \cosh (a \tau)=0 .
$$

Eliminating $\tau$ between (3.59) and (3.60), and putting $u=\tau \sqrt{a}$, we find that the caustic can also be written in parametric form as follows:

$$
\begin{aligned}
(a x)^{2}= & \frac{1}{2(\tanh u-u)^{2}}\left\{-\left(2 u^{3}(\tanh u-u)+\delta^{2} u^{4} \tanh ^{2} u\right)\right. \\
& \left.+\delta u^{3}\left(4+\delta^{2} u^{2}\right)^{1 / 2}(\tanh u)^{3 / 2}(\tanh u-g(u))^{1 / 2}\right\} \\
a z= & a z_{0}+\frac{1}{u}\left(u^{2}-(\beta x)^{2}\right)^{1 / 2} \sinh u, \quad u \geq u_{0},
\end{aligned}
$$


where $u_{0}$ is the root of the equation $g(u)=\tanh u$ with $g(u)=\frac{4 u}{4+\left(a z_{0}\right)^{2} u^{2}}, \delta=a z_{0}$. By differentiating the equations (3.61), we can show that $\left(x\left(u_{0}\right), z\left(u_{0}\right)\right)$ corresponds to a cusp singularity.

Motivated by the above examples, in order to study the GCP when $\eta^{\prime}(z)$ has discontinuities, we distinguish two cases according to whether there are or are not rays tangent to the interface. We assume that there exists a point $z=c$ and smooth functions $\eta_{i}(z)$ $(i=1,2)$ such that $\eta_{1}(z)$ is smooth on $(-\infty, c+\varepsilon)$ and $\eta_{2}(z)$ is smooth on $(c-\varepsilon, \infty)$ for sufficiently small $\varepsilon>0$. Moreover, we assume that $\eta_{1}(c)=\eta_{2}(c)$ and $\eta_{1}^{\prime}(c) \neq \eta_{2}^{\prime}(c)$. We consider the following three subsets of $\mathbb{R}^{2}$ :

$$
\mathbb{R}_{<c+\varepsilon}^{2}=\{(x, z) \mid z<c+\varepsilon\}, \quad \mathbb{R}_{>c-\varepsilon}^{2}=\{(x, z) \mid z>c-\varepsilon\} \quad \text { and } \quad \mathbb{R}_{c}^{2}=\{z=c\},
$$

and the corresponding subspaces of the phase space $\mathbb{R}^{5}$

$$
\begin{aligned}
\mathbb{R}_{<c+\varepsilon}^{4} & =\{(x, z, p, q) \mid z<c+\varepsilon\}, \\
\mathbb{R}_{>c-\varepsilon}^{4} & =\{(x, z, p, q) \mid z>c-\varepsilon\}, \\
\mathbb{R}_{c}^{4} & =\{(x, z, p, q) \mid z=c\} .
\end{aligned}
$$

We denote $H_{i}(x, z, p, q)=\frac{1}{2}\left(p^{2}+q^{2}-\eta_{i}^{2}(z)\right)$, and we also consider the following sets:

$$
\begin{aligned}
\mathcal{H}_{<c} & =H_{1}^{-1}(0) \cap\{(x, z, p, q) \mid z<c\}, \\
\mathcal{H}_{<c+\varepsilon} & =H_{1}^{-1}(0) \cap \mathbb{R}_{<c+\varepsilon}^{4}, \\
\mathcal{H}_{>c-\varepsilon} & =H_{2}^{-1}(0) \cap \mathbb{R}_{>c-\varepsilon}^{4}, \\
\mathcal{H}_{c} & =H_{1}^{-1}(0) \cap \mathbb{R}_{c}^{4}=H_{2}^{-1}(0) \cap \mathbb{R}_{c}^{4} .
\end{aligned}
$$

In each region $\mathbb{R}_{>c+\varepsilon}^{4}$ or $\mathbb{R}_{<c-\varepsilon}^{4}$ we have the same framework as in the smooth refraction index case considered in Sec. 3.2. Let us also assume that the initial data of the GCP are located in $\mathcal{H}_{<c}$.

Thus, we consider a Legendrian curve $\ell: I \rightarrow \mathcal{H}_{<c}$ as initial data for the GCP. As in the previous section, a ray starting from $\ell$ is given by the characteristic equations (cf. Eq. (C) in Sec. 3.1)

$$
\left\{\begin{array}{l}
\dot{x}_{1}=p_{1}, \quad \dot{z}_{1}=q_{1}, \quad \dot{p}_{1}=0 \dot{q}_{1}=\eta_{1}(z) \eta_{1}^{\prime}(z), \\
x_{1}(0, \theta)=x(\theta), \quad z_{1}(0, \theta)=z(\theta), \quad p_{1}(0, \theta)=p(\theta), \quad q_{1}(0, \theta)=q(\theta) .
\end{array}\right.
$$

The solution in $\mathcal{H}_{<c+\varepsilon}$ is

$$
x_{1}(\tau, \theta)=p(\theta) \tau+x(\theta), \quad z_{1}(\tau, \theta), \quad p_{1}(\tau, \theta)=p(\theta), \quad q_{1}(\tau, \theta)=\dot{z}_{1}(\tau, \theta)
$$

where

$$
\ddot{z}_{1}(\tau, \theta)=\eta_{1}\left(z_{1}(\tau, \theta)\right) \eta_{1}^{\prime}\left(z_{1}(\tau, \theta)\right), \quad z_{1}(0, \theta)=z(\theta) \quad \text { and } \quad \dot{z}_{1}(0, \theta)=q(\theta) .
$$

If the ray $R_{\theta}(\tau)$ reaches the line $\mathbb{R}_{c}^{2}$ at the time $\tau_{0}$, we have

$$
x_{1}\left(\tau_{0}, \theta\right)=p(\theta) \tau_{0}+x(\theta), \quad z_{1}\left(\tau_{0}, \theta\right)=c, \quad p_{1}\left(\tau_{0}, \theta\right)=p(\theta), \quad q_{1}\left(\tau_{0}, \theta\right)=\dot{z}_{1}\left(\tau_{0}, \theta\right) .
$$


Since we have the relation $p^{2}(\theta)+\left(\dot{z}_{1}\right)^{2}\left(\tau_{0}, \theta\right)=\left(\eta_{1}\right)^{2}(c)=\left(\eta_{2}\right)^{2}(c)$, we can start the ray from $\mathbb{R}_{c}^{2}$ as the solution of the characteristic equations:

$$
\left\{\begin{array}{l}
\dot{x}_{2}=p_{2}, \quad \dot{z}_{2}=q_{2}, \quad \dot{p}_{2}=0, \quad \dot{q}_{2}=\eta_{2}\left(z_{2}\right) \eta_{2}^{\prime}\left(z_{2}\right), \\
x_{2}(0, \theta)=p(\theta) \tau_{0}+x(\theta), \quad z_{2}(0, \theta)=c, \quad p_{2}(0, \theta)=p(\theta), \quad q_{2}(0, \theta)=\dot{z}_{1}\left(\tau_{0}, \theta\right) .
\end{array}\right.
$$

The solution in $\mathcal{H}_{>c-\varepsilon}$ is

$$
x_{2}(\sigma, \theta)=p(\theta)\left(\tau_{0}+\sigma\right)+x(\theta), \quad z_{2}(\sigma, \theta), \quad p_{2}(\sigma, \theta)=p(\theta), \quad q_{2}(\sigma, \theta)=\dot{z}_{2}(\sigma, \theta),
$$

where

$$
\ddot{z}_{2}(\sigma, \theta)=\eta_{2}\left(z_{2}(\sigma, \theta)\right) \eta_{2}^{\prime}\left(z_{2}(\sigma, \theta)\right), \quad z_{2}(0, \theta)=c, \quad \dot{z}_{2}(0, \theta)=\dot{z}_{1}(\tau, \theta) .
$$

Since $\dot{z}_{2}(0, \theta)=\dot{z}_{1}\left(\tau_{0}, \theta\right)$, the rays have a continuous change of slope across $\mathbb{R}_{c}^{2}$.

By the arguments in Sec. 3.1, we construct the Lagrangian solution $\Gamma_{1}: \mathbb{R}_{+} \times I \rightarrow$ $\mathcal{H}_{<c+\varepsilon}$ for the initial data $\ell: I \rightarrow \mathcal{H}_{<c}$. According to Theorem 3.2, the appearing caustics have only fold or cusp singularities for generic initial data $\ell$.

We now distinguish the following two cases.

Case A. All rays starting from $\ell$ are not tangent to the line $\mathbb{R}_{c}^{2}$.

Our assumption means that $\dot{z}_{1}(\tau, \theta) \neq 0$ for $z_{1}(\tau, \theta)=c$. It follows from the implicit function theorem that there exists (at least locally) a smooth function $\tau=\tau(\theta, c)$ such that $z_{1}(\tau(\theta, c), \theta)=c$. We now consider the local parameterized curve in $\mathcal{H}_{>c-\varepsilon}$ given by

$$
\ell_{c}(\theta)=\left(p(\theta) \tau(\theta, c)+x(\theta), c, p(\theta), \dot{z}_{1}(\tau(\theta, c), \theta)\right) .
$$

We can easily show that

$$
\ell_{c}^{*} \alpha=\eta_{1}(c)^{2} \frac{d \tau}{d \theta} d \theta=d\left(\eta_{1}(c)^{2} \tau(\theta, c)\right)=d\left(\eta_{2}(c)^{2} \tau(\theta, c)\right)
$$

By a direct calculation we have

$$
\begin{aligned}
\ell_{c}^{\prime}(\theta)=\left(\begin{array}{ll}
p(\theta) & \dot{z}_{1}(\tau(\theta, c), \theta) \\
p^{\prime}(\theta) \tau(\theta, c)+x^{\prime}(\theta) & \frac{\partial z}{\partial \theta}(\tau(\theta, c), \theta)
\end{array} \mid\right. \\
\dot{z}_{1}(\tau(\theta, c), \theta)
\end{aligned}
$$

If $\ell_{c}^{\prime}(\theta)=0$, then the rank of the Jacobi matrix of the Lagrangian solution $\Gamma_{1}$ in $\mathcal{H}_{<c+\varepsilon}$ is at most 1 . This contradicts the fact that $\Gamma_{1}$ is an immersion. Therefore, we have: $\ell_{c}^{\prime}(\theta) \neq 0$, for any $\theta \in I$. We also have $X_{H_{2}} \circ \ell_{c}(\theta)=\left(p(\theta), \dot{z}_{1}(\tau(\theta, c), \theta), 0, \eta_{2}(c)\right)$. By means of the assumption that $\dot{z}_{1}(\tau(\theta, c), \theta) \neq 0$ we have that $X_{H_{2}} \circ \ell_{c}(\theta)$ and $\ell_{c}^{\prime}(\theta)$ are linearly independent. 
We can construct the Lagrangian solution $\Gamma_{2}$ in $\mathcal{H}_{>c-\varepsilon}$ with initial submanifold $\ell_{c} \subset$ $\mathcal{H}_{>c-\varepsilon}$. Note that $\ell_{c}$ is not a Legendrian submanifold in $\mathcal{H}_{>c-\varepsilon}$. However, since $\ell_{c}^{*} \Theta=0$ and the Hamiltonian flow preserves the canonical two-form $\Theta$, we can apply the method of characteristics with the initial condition $\ell_{c}$.

Moreover, we also construct $\Gamma_{2}$ for opposite directed flows. It follows that we have the local parameterization of the Lagrangian solution

$$
\Gamma_{2}(\sigma, \theta)=\left(p(\theta)(\tau(\theta, c)+\sigma)+x(\theta), z_{2}(\sigma, \theta), p(\theta), \dot{z}_{2}(\sigma, \theta)\right),
$$

where

$$
z_{1}(\tau(\theta, c), \theta)=c=z_{2}(0, \theta), \quad \ddot{z}_{2}(\sigma, \theta)=\eta_{2}\left(z_{2}(\sigma, \theta)\right) \eta_{2}^{\prime}\left(z_{2}(\sigma, \theta)\right)
$$

and

$$
\dot{z}_{2}(0, \theta)=\dot{z}_{1}(\tau(\theta, c), \theta)
$$

Therefore we have two Lagrangian submanifolds $\Gamma_{1}, \Gamma_{2}$ in the neighborhood of $\mathbb{R}_{c}^{4}$ in $\mathbb{R}^{4}$. Since

$$
\frac{\partial z_{2}}{\partial \theta}(0, \theta)=\frac{\partial z_{1}}{\partial \theta}(\tau(\theta, c), \theta)=0
$$

the Jacobian matrix of $\Gamma_{2}$ at a point in $\mathbb{R}_{c}^{2}$ is

$$
\left(\begin{array}{cccc}
p(\theta) & \dot{z}_{2}(0, \theta) & 0 & \ddot{z}_{2}(0, \theta) \\
p^{\prime}(\theta) \tau(\theta, c)+x^{\prime}(\theta) & 0 & p^{\prime}(\theta) & \frac{\partial \dot{z}_{2}}{\partial \theta}(0, \theta)
\end{array}\right) .
$$

By the calculation of the Jacobian matrix for $\Gamma_{1}$ in Sec. 3.1, this matrix is the same as the Jacobian matrix of $\Gamma_{2}$ at the same point in $\mathbb{R}_{c}^{2}$. Then, we have the following theorem.

Theorem 3.4. (1) For a generic initial Legendrian curve $\ell: I \rightarrow \mathcal{H}_{<c}$, the caustics of $\Gamma_{1}$ have only cusp or fold singularities. Moreover, cusps do not appear on $\mathbb{R}_{c}^{2}$.

(2) If a point $\pi \circ \Gamma_{1}\left(\tau_{0}, \theta_{0}\right)=\pi \circ \Gamma_{2}\left(0, \theta_{0}\right) \in \mathbb{R}_{c}^{2}$ is a fold point of $\pi \circ \Gamma_{1}$, then it is also a fold point of $\pi \circ \Gamma_{2}$.

Proof. The first part of the assertion (1) follows from Theorem 3.2. If cusps appear on $\mathbb{R}_{c}^{2}$ we can apply Bogaevskiı̌'s theorem [BO] to move away cusps from $\mathbb{R}_{c}^{2}$ by a small perturbation of the initial Legendrian curve $\ell$ (or $\ell_{c}$ ). For the assertion (2), we have shown that the considered point is a singular point of $\pi \circ \Gamma_{1}$ if and only if it is a singular point of $\pi \circ \Gamma_{2}$. Since $\pi \circ \Gamma_{1}$ and $\pi \circ \Gamma_{2}$ have common Jacobian matrix

$$
\left(\begin{array}{cc}
p\left(\theta_{0}\right) & \dot{z}_{2}(0, \theta) \\
p^{\prime}\left(\theta_{0}\right) \tau\left(\theta_{0}, c\right)+x^{\prime}(\theta) & 0
\end{array}\right)
$$

we have

$$
\text { Image } \alpha\left(\pi \circ \Gamma_{1}\right)_{\left(\tau_{0}, \theta_{0}\right)}=\text { Image } \alpha\left(\pi \circ \Gamma_{2}\right)_{\left(0, \theta_{0}\right)} \text {. }
$$

By the assumption the dimension of Image $\alpha\left(\pi \circ \Gamma_{1}\right)_{\left(\tau_{0}, \theta_{0}\right)}$ is one. It follows that $\pi \circ \Gamma_{2}$ has a fold point at $\left(0, \theta_{0}\right)$.

The above theorem asserts that if the fold locus approaches $\mathbb{R}_{c}$ transversally, then the fold locus continues to $\mathbb{R}_{>c}^{2}=\{(x, z) \mid z>c\}$. The natural conjecture is that $\Gamma_{2}$ has only cusps and folds for a generic initial Legendrian curve $\ell: I \rightarrow \mathcal{H}_{<c}$. Kazarian [KA] asserts that if a caustic of $\Gamma_{1}$ has only cusps or folds, then the caustics of $\Gamma_{2}$ have only cusps 
or folds under a small perturbation of $\Gamma_{2}$ in the space of all Lagrangian submanifolds. However, the above conjecture is still open.

Case B. There exists a ray $R_{\theta_{0}}(\tau)$ starting from $\ell$ such that $R_{\theta_{0}}(\tau)$ is tangent to the line $\mathbb{R}_{c}^{2}$.

Many complicated phenomena occur in this case. We consider the Lagrangian solution $\Gamma_{1}: R_{+} \times I \rightarrow \mathcal{H}_{<c+\varepsilon}$ for generic initial data $\ell: I \rightarrow \mathcal{H}_{<c}$. So caustics of $\Gamma_{1}$ have only folds or cusps. We assume that there exists $\left(\tau_{0}, \theta_{0}\right) \in \mathbb{R}_{+} \times I$ such that $R_{\theta_{0}}^{1}(\tau)$ is tangent to the line $\mathbb{R}_{c}^{2}$ at the point $\mathbf{q}_{0}=\left(p\left(\theta_{0}\right) \tau_{0}+x\left(\theta_{0}\right), c\right)$. This means that $z_{1}\left(\tau_{0}, \theta_{0}\right)=c$ and $\dot{z}_{1}\left(\tau_{0}, \theta_{0}\right)=0$. We can assert again that cusps are not located on the line $\mathbb{R}_{c}^{2}$ for generic initial data. Without loss of generality we may assume that all rays $R_{\theta}^{1}(\tau)$ for $\theta>\theta_{0}$ are not tangent to the line $\mathbb{R}_{c}$, that is, the point $\theta_{0}$ divides the interval $I$ into two parts $I_{<\theta_{0}}$ and $I_{>\theta_{0}}$.

Since, if we restrict the initial data $\ell$ to $\theta_{0}=\ell \mid I_{>\theta_{0}}$ we are in Case A, we may also assume that caustics do not appear at the point $\mathbf{q}_{0}$. If a caustic appears at the point $\mathbf{q}_{\mathbf{0}}$, we may move it away from qo by a small perturbation of $\ell$ (or $\ell_{c}$ ). Moreover, we consider the case where $\eta_{1}^{\prime}(z)<0$ for any $z<c+\varepsilon$. In this case, all rays $R_{\theta}^{1}(\tau)$ for $\theta<\theta_{0}$ do not reach the line $\mathbb{R}_{c}^{2}$. We also assume that $\eta_{1}^{\prime}(c) \neq 0$ and $\eta_{2}^{\prime}(c) \neq 0$.

We now further consider two cases as follows.

Case B1. $\eta_{2}^{\prime}\left(z_{2}\right)<0$.

In this case we have $\ddot{z}_{2}=\eta_{2}\left(z_{2}\right) \eta_{2}^{\prime}\left(z_{2}\right)<0$. Then the rays starting from $\mathbb{R}_{c}^{2}$ for $\theta>\theta_{0}$ return to $\mathbb{R}_{c}^{2}$ for $(x, c)$ with $x>p\left(\theta_{0}\right) \tau_{0}+x\left(\theta_{0}\right)$. We have no shadow zones (i.e., areas that are not covered by characteristics) around the point $\mathbf{q}_{\mathbf{0}}$. For this case, the situation is the same as that of Case A described by Theorem 3.4.

Case B2. $\eta_{2}^{\prime}\left(z_{2}\right)>0$.

Then the ray reaching the point $\mathbf{q}_{0}$ bifurcates so that one branch, denoted by $R_{\theta_{0}}^{1}(\tau)$, continues into $\mathbb{R}_{<c}^{2}$ and another branch, denoted by $R_{\theta_{0}}^{2}$, enters the region $\mathbb{R}_{>c}^{2}$. W have a shadow zone around the point $\mathbf{q}_{0}$ (see Fig. 1 ). We call the rays $R_{\theta_{0}}^{1}, R_{\theta_{0}}^{2}$ boundary rays.

If we consider the restriction of the Lagrangian solution $\Gamma_{1}$ to $\mathbb{R}_{+} \times I_{>\theta_{0}}$, then only folds approach $\left(\tau_{0}, \theta_{0}\right) \in \mathbb{R}_{+} \times\left\{\theta_{0}\right\}$. Indeed, if the cusp point appears at the point $\left(\tau_{0}, \theta_{0}\right) \in \mathbb{R}_{+} \times\left\{\theta_{0}\right\}$, we can move it away from $\mathbb{R}_{+} \times\left\{\theta_{0}\right\}$ by exactly the same arguments as those in the proof of Theorem 3.4. If we consider the restriction of $\Gamma_{1}$ to $\mathbb{R}_{+} \times I_{\leq \theta_{0}}$, then we have a domain with a boundary. New types of caustics might appear at a point of $R_{\theta_{0}}^{1}$, for $\tau>\tau_{0}$, which are called caustics with boundary (or edge caustics) (see Fig. 2).

As in the ordinary theory of Lagrangian singularities (cf. Arnold et al $[\mathrm{AVH}]$ ), a Lagrangian manifold with boundary is given locally by a generating family $F\left(x, y, \lambda_{1}, \lambda_{2}\right)$. Then, we have

$$
\begin{gathered}
L_{ \pm}=\left\{\left(\lambda_{1}, \lambda_{2}, \kappa_{1}, \kappa_{2}\right) \in T^{*} \mathbb{R}^{2} \mid \exists(x, y), F_{x}=F_{y}=0, \kappa_{1}=F_{\lambda_{1}}, \kappa_{2}=F_{\lambda_{2}}, \pm x \geq 0\right\} \\
\partial L=\left\{\left(\lambda_{1}, \lambda_{2}, \kappa_{1}, \kappa_{2}\right) \in L \mid x=0\right\} .
\end{gathered}
$$

We consider the equivalence relation among Legendrian manifolds with boundary. The natural equivalence relation is the Lagrangian equivalence preserving the boundary. 


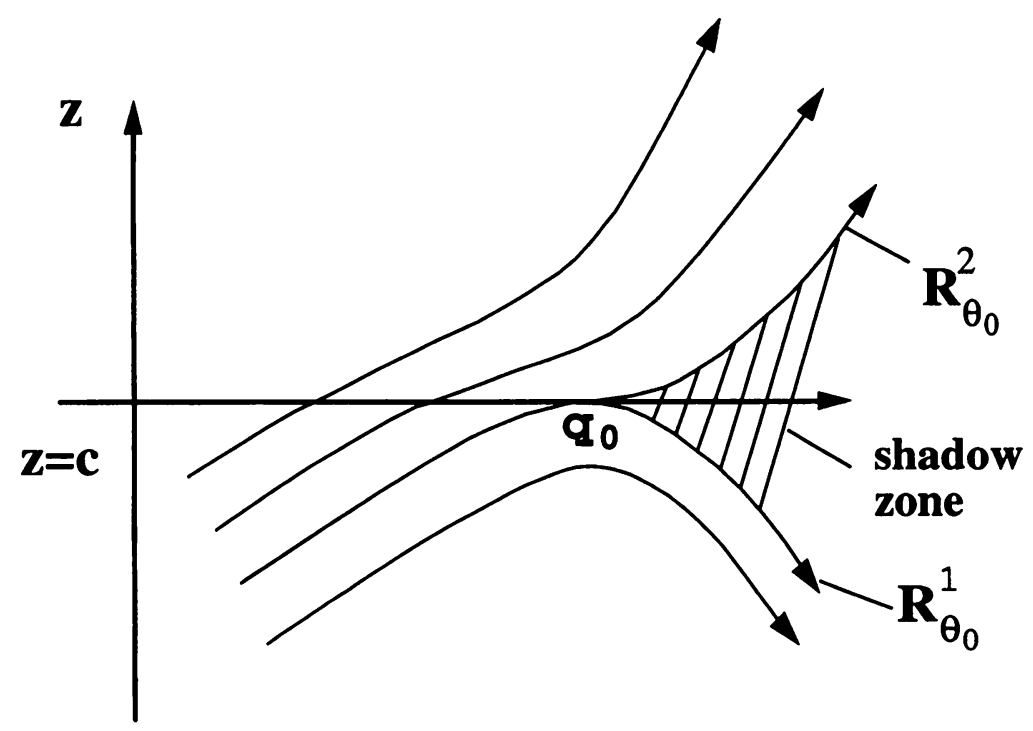

FIG. 2. Refracted rays and shadow boundary

There is a corresponding equivalence relation among generating families and we have the following classification theorem (cf. Arnold [AVH] (17.4)).

THEOREM 3.5. The generic singularities of caustics with boundary at boundary points in $\mathbb{R}^{2}$ are as follows:

$B_{2}=C_{2}$ with generating family $\pm x^{2} \pm y^{2}+\lambda_{1} x$

$B_{3}$ with generating family $x^{3} \pm y^{2}+\lambda_{1} x^{2}+\lambda_{2} x$,

$C_{3}$ with generating family $x y+y^{3}+\lambda_{1} y^{2}+\lambda_{2} y$.

The boundary corresponds to the set $\{x=0\}$.

For example, in the case $B_{2}=C_{2}$ we have $L_{ \pm}=\left\{\left(\mp 2 x, \lambda_{2}, x, 0\right) \mid \pm x \geq 0, \lambda_{2} \in \mathbb{R}\right\}$ and $\partial L_{ \pm}=\left\{\left(0, \lambda_{2}, 0,0\right) \mid \lambda_{2} \in \mathbb{R}\right\}$. Therefore, the boundary on $\mathbb{R}^{2}$ is the set $\pi\left(\partial L_{ \pm}\right)$ which is the $\lambda_{2}$-axis. Such boundary points are those of the boundary ray that separates the illuminated zone from the shadow zone of Fig. 1. For the $B_{3}$ case, $L_{ \pm}=\left\{\left(\lambda_{1},-3 x^{2}-\right.\right.$ $\left.\left.2 \lambda_{1} x, x^{2}, x\right) \mid \pm x \geq 0, \lambda_{1} \in \mathbb{R}\right\}$. Therefore, $\partial L_{ \pm}=\left\{\left(\lambda_{1}, 0,0,0\right) \mid \lambda_{1} \in \mathbb{R}\right\}$ and the set $\pi\left(\partial L_{ \pm}\right)$is the $\lambda_{1}$-axis. The caustic of $L_{ \pm}$is the set $\left\{\left(-3 x, 3 x^{2}\right) \mid x \in \mathbb{R}\right\}$. So, $B_{3}$ describes the situation of a caustic with boundary, that is, the Lagrangian submanifold around the point $\left(x_{b}, z_{b}\right)$ of Fig. 3. For the $C_{3}$ case, $L_{ \pm}=\left\{\left(\lambda_{1},-x, 0,0\right) \mid \pm x \geq 0, \lambda_{1} \in \mathbb{R}\right\}$ and $\partial L_{ \pm}=\left\{\left(\lambda_{1}, 0,0,0\right) \mid \lambda_{1} \in \mathbb{R}\right\}$ and the set $\pi\left(\partial L_{ \pm}\right)$is the $\lambda_{1}$-axis.

Case B2 has some analogy with the case where $z=c$ is considered an impedance boundary. Then, different kinds of caustics with boundary can appear. We look for a geometric solution of the eikonal equation in a restricted domain where the rays meeting the boundary reflect according to Snell's law (see, e.g., [TC], [CMP]). The presence of boundaries gives rise to geometric entities such as boundary rays, caustics with boundary and shadow zones. 


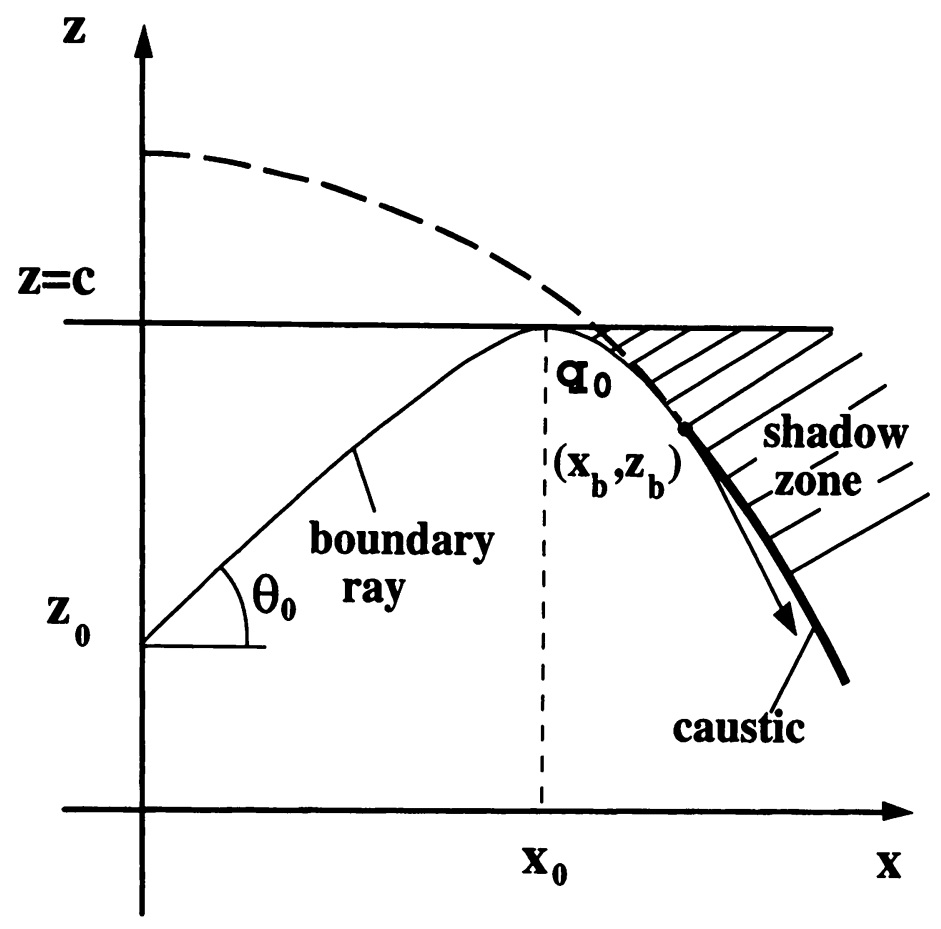

FIG. 3. Boundary ray and shadow zone

Let us study more Example 1, where we are now assuming that $z_{0}>0$ and that we have a boundary at $z=c, c<d$.

Then, the ray shot with initial angle $\theta=\theta_{0}=\arcsin \left(\sqrt{1-z_{0} / c}\right)$ hits the boundary $z=c$ at $x_{0}=2 \sqrt{z_{0}\left(c-z_{0}\right)}$, and it is tangent to the caustic at the point (see Fig. 2)

$$
x_{b}=2 c\left(\frac{z_{0} / c}{1-z_{0} / c}\right)^{1 / 2}, \quad z_{b}=c\left(2+\frac{z_{0}}{c}-\frac{1}{1-z_{0} / c}\right) .
$$

Consequently all the rays shot with $\theta>\theta_{0}$ are reflected from the boundary $z=c$, while all the others turn downwards before hitting the boundary and form a caustic, which is the part of the parabola (3.31) with $z<z_{b}$. The two sets of rays are separated by the ray shot at $\theta_{0}$ that meets the caustic at its end point $\left(x_{b}, z_{b}\right)$. This ray together with the caustic form the boundary of the shadow zone in which no ray can penetrate, and for this we call this special ray the boundary ray and the appearing caustic the boundary caustic.

The geometry of the rays in this case is different from that of case (B2). Following Scherback [SC] (see also [TS1], [TS2]), to define the boundary caustics we need the notion of a Lagrangian pair $\left(L_{ \pm}, L_{0}\right)$. In this case we can also construct (at least locally) the 
generating family $F$ for any Lagrangian pair $\left(L_{ \pm}, L_{0}\right)$ as follows:

$$
\begin{aligned}
& L_{ \pm}=\left\{\left(\lambda_{1}, \lambda_{2}, \kappa_{1}, \kappa_{2}\right) \in T^{*} \mathbb{R}^{2} \mid \exists(x, y), F_{x}=F_{y}=0, \kappa_{1}=F_{\lambda_{1}}, \kappa_{2}=F_{\lambda_{2}}, \pm x \geq 0\right\} \\
& L_{0}=\left\{\left(\lambda_{1}, \lambda_{2}, \kappa_{1}, \kappa_{2}\right) \in T^{*} \mathbb{R}^{2} \mid \exists y, F_{y}\left(0, y, \lambda_{1}, \lambda_{2}\right)=0, \kappa_{1}=F_{\lambda_{1}}, \kappa_{2}=F_{\lambda_{2}}\right\},
\end{aligned}
$$

and $\pi\left(L_{ \pm} \cap L_{0}\right)$ consists of the boundary points that might include boundary caustics, where $\pi$ is the projection onto the $\left(\lambda_{1}, \lambda_{2}\right)$ space. We have the following theorem (see [SC], cf. [TS1], [TS2]).

THEOREM 3.6. The stable singularities of boundary caustics at boundary points in $\mathbb{R}^{2}$ are as follows:

$B_{2}=C_{2}$ with generating family $\pm x^{2} \pm y^{2}+\lambda_{1} x$

$B_{3}$ with generating family $x^{3} \pm y^{2}+\lambda_{1} x^{2}+\lambda_{2} x$,

$C_{3}$ with generating family $x y+y^{3}+\lambda_{1} y^{2}+\lambda_{2} y$.

The boundary corresponds to the set $\{x=0\}$.

For the case $B_{2}=C_{2}$ we have

$$
L_{ \pm}=\left\{\left(\mp 2 x, \lambda_{2}, x, 0\right) \mid \pm x \geq 0, \lambda_{2} \in \mathbb{R}\right\}, \quad L_{0}=\left\{\left(\lambda_{1}, \lambda_{2}, 0,0\right) \mid \lambda_{1}, \lambda_{2} \in \mathbb{R}\right\} .
$$

Therefore, $L_{ \pm} \cap L_{0}=\left\{\left(0, \lambda_{2}, 0,0\right) \mid \lambda_{2} \in \mathbb{R}\right\}$ and the set $\pi\left(L \cap L_{0}\right)$ is the $\lambda_{2}$-axis. Such boundary points are those of the boundary ray that separates the illuminated zone from the shadow zone of Fig. 2.

For the $B_{3}$ case we have

$$
L_{ \pm}=\left\{\left(\lambda_{1},-3 x^{2}-2 \lambda_{1} x, x^{2}, x\right) \mid \pm x \geq 0, \lambda_{1} \in \mathbb{R}\right\}, \quad L_{0}=\left\{\left(\lambda_{1}, \lambda_{2}, 0,0\right) \mid \lambda_{1}, \lambda_{2} \in \mathbb{R}\right\} .
$$

Therefore, $L_{ \pm} \cap L_{0}=\left\{\left(\lambda_{1}, 0,0,0\right) \mid \lambda_{1} \in \mathbb{R}\right\}$ and the set $\pi\left(L_{ \pm} \cap L_{0}\right)$ is the $\lambda_{1}$-axis. The caustic of $L_{ \pm}$is the set $\left\{\left(-3 x, 3 x^{2}\right) \mid x \in \mathbb{R}\right\}$. So, $B_{3}$ describes the case of a boundary ray meeting a boundary caustic which, around the point $\left(x_{b}, z_{b}\right)$, depicts the geometry of Fig. 3 ( $z=c$ is considered a hard boundary).

Finally, for the $C_{3}$ case we have

$$
\begin{aligned}
L_{ \pm} & =\left\{\left(\lambda_{1},-x, 0,0\right) \mid \pm x \geq 0, \lambda_{1} \in \mathbb{R}\right\}, \\
L_{0} & \left.=\left\{\left(\lambda_{1}-3 y^{2}-2 y \lambda_{1}, y^{2}, y\right)\right) \mid y \in \mathbb{R}, \lambda_{1} \in \mathbb{R}\right\} .
\end{aligned}
$$

Therefore, $L_{ \pm} \cap L_{0}=\left\{\left(\lambda_{1}, 0,0,0\right) \mid \lambda_{1} \in \mathbb{R}\right\}$ and the set $\pi\left(L_{ \pm} \cap L_{0}\right)$ is the $\lambda_{1}$-axis. The caustic of $L_{0}$ is the set $\left\{\left(-3 y, 2 y^{2}\right) \mid y \in \mathbb{R}\right\}$.

\section{REFERENCES}

[ABG] R. Abgrall and J. D. Benamou, Big ray tracing and eikonal solver on unstructured grids: Application to the computation of a multivalued travel-time field in the marmousi model, INRIA Tech. Rep. 3019 (1997)

[AK] G. Avila and J. Keller, The high-frequency asymptotic field of a point source in an inhomogeneous medium, Comm. Pure Appl. Math. XVI, 363-381 (1963)

[AN] V. I. Arnol'd and S. P. Novikov (Eds.), Dynamical Systems IV, Symplectic Geometry, Encyclopedia of Math. Sci., Vol. 4, Springer, 1985

[AND] V. B. Andeev, A. V. Demin, Yu. A. Kravtsov, M. V. Tinin, and A. P. Yarygin, The interferential integral method (a review), Radiophys. Quantum Electron. 31(N11), 907-921 (1988)

[ARN1] J. M. Arnold, Oscillatory integral theory for uniform representation of wave functions, Rad. Sci. 17(5), 1181-1191 (1982) 
[ARN2] J. M. Arnold, Spectral synthesis of uniform wavefunctions, Wave Motion 8, 135-150 (1986)

[AVH] V. I. Arnol'd, A. N. Varchenko, and S. M. Guisern-Zade, Singularities of Differentiable Maps, Vol. 1, Birkhäuser-Verlag, Basel, 1985

[BEN] J. D. Benamou, Big ray tracing: Multivalued travel time field computation using viscosity solutions of the eikonal equation, J. Comput. Phys. 128, 463-474 (1996)

[B] V. B. Babich, The short wave asymptotic form of the solution for the problem of a point source in an inhomogeneous medium, USSR J. Comput. Math. Phys. 5(5), 949-951 (1965)

[BB] V. B. Babich and V. S. Buldyrev, Short-Wavelength Diffraction Theory. Asymptotic Methods, Springer-Verlag, Berlin-Heidelberg, 1991

[BKP] J. D. Benamou, T. Katsaounis, and B. Perthame, High frequency Helmholtz equation, geometrical optics and particle methods, preprint (1998)

[BR] L. M. Brekhovskikh, Waves in Layered Media, Academic Press, New York, 1980

[BH] N. Bleistein and R. Handelsman, Asymptotic Expansions of Integrals, Dover Publications Inc., New York, 1986

[BK] V. M. Babich and N. Y. Kirpichnikova, The Boundary-Layer Method in Diffraction Problems, Springer-Verlag, Berlin-Heidelberg, 1979

[BO] I. A. Bogaevskiǔ, Perestroikas of fronts in evolutionary families, Proc. of the Steklov Inst. of Math. 209, 57-72 (1995)

[CK] D. Colton and R. Kress, Inverse Acoustic and Electromagnetic Scattering, Springer-Verlag, New York, 1992

[CMP] V. Cĕrvenỳ, I. A. Molotkov, and I. Pšenčik, Ray Method in Seismology, Univerzita Karlova, Praha, 1977

[DUI] J. J. Duistermaat, Oscillatory integrals, Lagrangian immersions and unfolding of singularities, Comm. Pure Appl. Math. XXVII, 207-281 (1974)

[FEO] E. Fatemi, B. Engquist, and S. Osher, Numerical solution of the high frequency asymptotic expansion for the scalar wave equation, J. Comput. Phys. 120(1), 145-155 (1995)

[GA] Yu. L. Gazaryan, On the Ray Approximation near Nonsingular Caustics, Dynamic Theory of Seismic Wave Propagation 5, LGU Press, Leningrad, 1961, pp. 73-92

[GS] V. Guillemin and S. Sternberg, Geometric Asymptotics, Mathematical Surveys, No. 14, American Mathematical Society, Providence, RI, 1977

[GSC] V. Guillemin and D. Schaeffer, Remarks on a paper of D. Ludwig, Bull. Amer. Math. Soc. 79(2), 382-385 (1973)

[HA] A. Hanyga, Canonical functions of asymptotic diffraction theory associated with symplectic singularities, Symplectic Singularities and Geometry of Gauge Fields, Banach Center Publications, Volume 36, Institute of Mathematics, Polish Academy of Sciences, Warszawa, 1997, pp. 57-71

[HS] A. Hanyga and M. Seredyńska, Diffraction of pulses in the vicinity of simple caustics and caustic cusps, Wave Motion 14, 101-121 (1991)

[HO1] L. Hörmander, The Analysis of Linear Partial Differential Operators I, Springer-Verlag, New York, 1983

[HO2] L. Hörmander, Fourier integral operators I, Acta Math. 127 (1971)

[IZ] S. Izumiya, Perestroikas of optical wave fronts and graphlike Legendrian unfoldings, J. Differential Geometry 38, 485-500 (1993)

[JA] K. Jänich, Caustics and catastrophes, Math. Ann. 209, 161-180 (1974)

[JO] D. S. Jones, High-frequency refraction and diffraction in general media, Philos. Trans. Roy. Soc. A 255, 341-387 (1963)

[KA] M. Kazarian, Caustics $D_{k}$ at points of interface between two media, Symplectic Geometry, London Math. Soc. Lecture Notes Series, vol. 192, 1993, pp. 115-125

[KKM] T. Katsaounis, G. T. Kossioris, and G. N. Makrakis, Computation of high frequency fields near caustics, Tech. Rep. 98.7, IACM-FORTH (1998)

[KO1] Yu. A. Kravtsov and Yu. I. Orlov, Caustics, Catastrophes and Wave Fields, Springer Series on Wave Phenomena, Springer-Verlag, Berlin, 1993

[KO2] Yu. A. Kravtsov and Yu. I. Orlov, Geometrical Optics of Inhomogeneous Media, Springer Series on Wave Phenomena, vol. 6, Springer-Verlag, Berlin, 1990

[KR] Yu. A. Kravtsov, Two new asymptotic methods in the theory of wave propagation in inhomogeneous media (review), Sov. Phys. Acoust. 14(1), 1-17 (1968)

[KU] V. V. Kucherenko, Quasiclassical asymptotics of a point-source function for the stationary Schrödinger equation, Theoret. Math. Phys. (English Translation) 1(3), 294-310 (1969) 
[LU] D. Ludwig, Uniform asymptotic expansions at a caustic, Comm. Pure Appl. Math. XIX, 215250 (1966)

[LY] V. V. Lychagin, Local classification of non-linear first order partial differential equations, Russian Math. Surveys 30, 105-175 (1975)

[MA1] V. P. Maslov, Operational Methods, Mir Publishers, Moscow, 1976

[MA2] V. P. Maslov, Theory of Perturbations and Asymptotic Methods, Dunod, Paris, 1972

[MF] V. P. Maslov and V. M. Fedoryuk, Semi-classical approximations in quantum mechanics, Contemp. Math. 5, D. Reidel, Dordrecht, 1981

[MSS] A. Mishchenko, V. Shatalov, and B. Sternin, Lagrangian Manifolds and the Maslov Operator, Springer-Verlag, Berlin-Heidelberg, 1990

[MY] O. M. Myasnichenko, Singularities of wave fronts on the interface between two media, St. Petersburg Math. J. 5, 789-807 (1994)

[RU] O. Runborg, Multiscale and Multiphase Methods for Wave Propagation, Doctoral Dissertation, Dept. Num. Anal. Comp. Sci., Roy. Inst. Techn. Stockholm, 1998

[SC] I. G. Scherbak, Boundary fronts and caustics and their metamorphosis, Singularities (J.-P. Brasselet, ed.), London Math. Soc. Lecture Note Series 201, 1994, pp. 363-373

[SYM] W. Symes, A slowness matching finite difference method for travel times beyond transmission caustics, Tech. Rep., Rice Univ., 1997

[TC] I. Tolstoy and C. S. Clay, Ocean Acoustics. Theory and Experiment in Underwater Sound, American Institute of Physics, New York, 1966

[TS1] T. Tsukada, Reticular Lagrangian singularities, Asian J. Math. 1, 572-622 (1997)

[TS2] T. Tsukada, Stability of optical caustics with r-corners, Hokkaido Math. J. 27, 633-650 (1998)

[V1] B. R. Vainberg, Asymptotic Methods in Equations of Mathematical Physics, Gordon and Breach, New York, 1989

[V2] B. R. Vainberg, Quasiclassical approximation in stationary scattering problems, Funct. Anal. Appl. 11, 247-257 (1977)

[WA] G. Wassermann, Stability of caustics, Math. Ann. 216, 43-50 (1975)

[WED] R. Weder, Spectral and Scattering Theory for Wave Propagation in Perturbed Stratified Media, Springer-Verlag, New York, 1991 